\title{
CYCLIC SIEVING FOR GENERALISED NON-CROSSING PARTITIONS ASSOCIATED WITH COMPLEX REFLECTION GROUPS OF EXCEPTIONAL TYPE
}

\author{
Christian KrattenthaleR ${ }^{\dagger}$ and Thomas W. MÜller ${ }^{\ddagger}$ \\ † Fakultät für Mathematik, Universität Wien, \\ Nordbergstraße 15, A-1090 Vienna, Austria. \\ WWW: http://www.mat.univie.ac.at/ kratt \\ $\ddagger$ School of Mathematical Sciences, \\ Queen Mary \& Westfield College, University of London, \\ Mile End Road, London E1 4NS, United Kingdom. \\ WWW: http://wWw.maths.qmw.ac.uk/ twm/
}

Dedicated to the memory of Herb Wilf

\begin{abstract}
We prove that the generalised non-crossing partitions associated with well-generated complex reflection groups of exceptional type obey two different cyclic sieving phenomena, as conjectured by Armstrong, and by Bessis and Reiner. The computational details are provided in the manuscript "Cyclic sieving for generalised non-crossing partitions associated with complex reflection groups of exceptional type — the details" [ar $\chi \mathrm{iv}: 1001.0030]$.
\end{abstract}

\section{INTRODUCTION}

In his memoir [2], Armstrong introduced generalised non-crossing partitions associated with finite (real) reflection groups, thereby embedding Kreweras' non-crossing partitions [22], Edelman's $m$-divisible non-crossing partitions [12], the non-crossing partitions associated with reflection groups due to Bessis [6] and Brady and Watt [10] into one uniform framework. Bessis and Reiner [9] observed that Armstrong's definition can be straightforwardly extended to well-generated complex reflection groups (see Section 2 for the precise definition). These generalised non-crossing partitions possess a wealth of beautiful properties, and they display deep and surprising relations to other combinatorial objects defined for reflection groups (such as the generalised cluster complex

2000 Mathematics Subject Classification. Primary 05E15; Secondary 05A10 05A15 05A18 06A07 $20 \mathrm{~F} 55$.

Key words and phrases. complex reflection groups, unitary reflection groups, $m$-divisible noncrossing partitions, generalised non-crossing partitions, Fuß-Catalan numbers, cyclic sieving.

${ }^{\dagger}$ Research partially supported by the Austrian Science Foundation FWF, grants Z130-N13 and S9607-N13, the latter in the framework of the National Research Network "Analytic Combinatorics and Probabilistic Number Theory."

${ }^{\ddagger}$ Research supported by the Austrian Science Foundation FWF, Lise Meitner grant M1201-N13. 
of Fomin and Reading [13, or the extended Shi arrangement and the geometric multichains of filters of Athanasiadis [4, 5]); see Armstrong's memoir [2] and the references given therein.

On the other hand, cyclic sieving is a phenomenon brought to light by Reiner, Stanton and White [30]. It extends the so-called "(-1)-phenomenon" of Stembridge [34, 35]. Cyclic sieving can be defined in three equivalent ways (cf. [30, Prop. 2.1]). The one which gives the name can be described as follows: given a set $S$ of combinatorial objects, an action on $S$ of a cyclic group $G=\langle g\rangle$ with generator $g$ of order $n$, and a polynomial $P(q)$ in $q$ with non-negative integer coefficients, we say that the triple $(S, P, G)$ exhibits the cyclic sieving phenomenon, if the number of elements of $S$ fixed by $g^{k}$ equals $P\left(e^{2 \pi i k / n}\right)$. In 30] it is shown that this phenomenon occurs in surprisingly many contexts, and several further instances have been discovered since then.

In [2, Conj. 5.4.7] (also appearing in [9, Conj. 6.4]) and [9, Conj. 6.5], Armstrong, respectively Bessis and Reiner, conjecture that generalised non-crossing partitions for irreducible well-generated complex reflection groups exhibit two different cyclic sieving phenomena (see Sections 3 and 7 for the precise statements).

According to the classification of these groups due to Shephard and Todd [32], there are two infinite families of irreducible well-generated complex reflection groups, namely the groups $G(d, 1, n)$ and $G(e, e, n)$, where $n, d, e$ are positive integers, and there are 26 exceptional groups. For the infinite families of types $G(d, 1, n)$ and $G(e, e, n)$, the two cyclic sieving conjectures follow from the results in [19].

The purpose of the present article is to present a proof of the cyclic sieving conjectures of Armstrong, and of Bessis and Reiner, for the 26 exceptional types, thus completing the proof of these conjectures. Since the generalised non-crossing partitions feature a parameter $m$, from the outset this is not a finite problem. Consequently, we first need several auxiliary results to reduce the conjectures for each of the 26 exceptional types to a finite problem. Subsequently, we use Stembridge's Maple package coxeter [36] and the GAP package CHEVIE [14, 28] to carry out the remaining finite computations. The details of these computations are provided in [21]. In the present paper, we content ourselves with exemplifying the necessary computations by going through some representative cases. It is interesting to observe that, for the verification of the type $E_{8}$ case, it is essential to use the decomposition numbers in the sense of [17, 18, 20] because, otherwise, the necessary computations would not be feasible in reasonable time with the currently available computer facilities. We point out that, for the special case where the aforementioned parameter $m$ is equal to 1 , the first cyclic sieving conjecture has been proved in a uniform fashion by Bessis and Reiner in [9]. (See [3] for a non-uniform — proof of cyclic sieving for non-crossing partitions associated with real reflection groups under the action of the so-called Kreweras map, a special case of the second cyclic sieving phenomenon discussed in the present paper.) The crucial result on which the proof of Bessis and Reiner is based is (5.5) below, and it plays an important role in our reduction of the conjectures for the 26 exceptional groups to a finite problem.

Our paper is organised as follows. In the next section, we recall the definition of generalised non-crossing partitions for well-generated complex reflection groups and of decomposition numbers in the sense of [17, 18, 20], and we review some basic facts. The first cyclic sieving conjecture is subsequently stated in Section 3. In Section 4, we outline an elementary proof that the $q$-Fuß-Catalan number, which is the polynomial $P$ in the cyclic sieving phenomena concerning the generalised non-crossing partitions 
for well-generated complex reflection groups, is always a polynomial with non-negative integer coefficients, as required by the definition of cyclic sieving. (Full details can be found in [21, Sec. 4]. The reader is referred to the first paragraph of Section 4 for comments on other approaches for establishing polynomiality with non-negative coefficients.) Section 5 contains the announced auxiliary results which, for the 26 exceptional types, allow a reduction of the conjecture to a finite problem. In Section 6, we discuss a few cases which, in a representative manner, demonstrate how to perform the remaining case-by-case verification of the conjecture. For full details, we refer the reader to [21, Sec. 6]. The second cyclic sieving conjecture is stated in Section 7. Section 8 contains the auxiliary results which, for the 26 exceptional types, allow a reduction of the conjecture to a finite problem, while in Section 9 we discuss some representative cases of the remaining case-by-case verification of the conjecture. Again, for full details we refer the reader to [21, Sec. 9].

\section{Preliminaries}

A complex reflection group is a group generated by (complex) reflections in $\mathbb{C}^{n}$. (Here, a reflection is a non-trivial element of $G L_{n}(\mathbb{C})$ which fixes a hyperplane pointwise and which has finite order.) We refer to [24] for an in-depth exposition of the theory complex reflection groups.

Shephard and Todd provided a complete classification of all finite complex reflection groups in [32] (see also [24, Ch. 8]). According to this classification, an arbitrary complex reflection group $W$ decomposes into a direct product of irreducible complex reflection groups, acting on mutually orthogonal subspaces of the complex vector space on which $W$ is acting. Moreover, the list of irreducible complex reflection groups consists of the infinite family of groups $G(m, p, n)$, where $m, p, n$ are positive integers, and 34 exceptional groups, denoted $G_{4}, G_{5}, \ldots, G_{37}$ by Shephard and Todd.

In this paper, we are only interested in finite complex reflection groups which are well-generated. A complex reflection group of rank $n$ is called well-generated if it is generated by $n$ reflections 1$]$ Well-generation can be equivalently characterised by a duality property due to Orlik and Solomon [29]. Namely, a complex reflection group of rank $n$ has two sets of distinguished integers $d_{1} \leq d_{2} \leq \cdots \leq d_{n}$ and $d_{1}^{*} \geq d_{2}^{*} \geq \cdots \geq d_{n}^{*}$, called its degrees and codegrees, respectively (see [24, p. 51 and Def. 10.27]). Orlik and Solomon observed, using case-by-case checking, that an irreducible complex reflection group $W$ of rank $n$ is well-generated if and only if its degrees and codegrees satisfy

$$
d_{i}+d_{i}^{*}=d_{n}
$$

for all $i=1,2, \ldots, n$. The reader is referred to [24, App. D.2] for a table of the degrees and codegrees of all irreducible complex reflection groups. Together with the classification of Shephard and Todd [32, this constitutes a classification of well-generated complex reflection groups: the irreducible well-generated complex reflection groups are

- the two infinite families $G(d, 1, n)$ and $G(e, e, n)$, where $d, e, n$ are positive integers,

- the exceptional groups $G_{4}, G_{5}, G_{6}, G_{8}, G_{9}, G_{10}, G_{14}, G_{16}, G_{17}, G_{18}, G_{20}, G_{21}$ of rank 2 ,

\footnotetext{
${ }^{1}$ We refer to [24, Def. 1.29] for the precise definition of "rank." Roughly speaking, the rank of a complex reflection group $W$ is the minimal $n$ such that $W$ can be realized as reflection group on $\mathbb{C}^{n}$.
} 
- the exceptional groups $G_{23}=H_{3}, G_{24}, G_{25}, G_{26}, G_{27}$ of rank 3,

- the exceptional groups $G_{28}=F_{4}, G_{29}, G_{30}=H_{4}, G_{32}$ of rank 4,

- the exceptional group $G_{33}$ of rank 5 ,

- the exceptional groups $G_{34}, G_{35}=E_{6}$ of rank 6 ,

- the exceptional group $G_{36}=E_{7}$ of rank 7 ,

- and the exceptional group $G_{37}=E_{8}$ of rank 8 .

In this list, we have made visible the groups $H_{3}, F_{4}, H_{4}, E_{6}, E_{7}, E_{8}$ which appear as exceptional groups in the classification of all irreducible real reflection groups (cf. [16]).

Let $W$ be a well-generated complex reflection group of rank $n$, and let $T \subseteq W$ denote the set of all (complex) reflections in the group. Let $\ell_{T}: W \rightarrow \mathbb{Z}$ denote the word length in terms of the generators $T$. This word length is called absolute length or reflection length. Furthermore, we define a partial order $\leq_{T}$ on $W$ by

$$
u \leq_{T} w \text { if and only if } \ell_{T}(w)=\ell_{T}(u)+\ell_{T}\left(u^{-1} w\right) .
$$

This partial order is called absolute order or reflection order. As is well-known and easy to see, the equation in (2.1) is equivalent to the statement that every shortest representation of $u$ by reflections occurs as an initial segment in some shortest product representation of $w$ by reflections.

Now fix a (generalised) Coxeter element² $c \in W$ and a positive integer $m$. The $m$-divisible non-crossing partitions $N C^{m}(W)$ are defined as the set

$$
\begin{aligned}
N C^{m}(W)=\left\{\left(w_{0} ; w_{1}, \ldots, w_{m}\right): w_{0} w_{1} \cdots\right. & w_{m}=c \text { and } \\
& \left.\quad \ell_{T}\left(w_{0}\right)+\ell_{T}\left(w_{1}\right)+\cdots+\ell_{T}\left(w_{m}\right)=\ell_{T}(c)\right\} .
\end{aligned}
$$

A partial order is defined on this set by

$$
\left(w_{0} ; w_{1}, \ldots, w_{m}\right) \leq\left(u_{0} ; u_{1}, \ldots, u_{m}\right) \quad \text { if and only if } \quad u_{i} \leq_{T} w_{i} \text { for } 1 \leq i \leq m .
$$

We have suppressed the dependence on $c$, since we understand this definition up to isomorphism of posets. To be more precise, it can be shown that any two Coxeter elements are related to each other by conjugation and (possibly) an automorphism on the field of complex numbers (see [33, Theorem 4.2] or [24, Cor. 11.25]), and hence the resulting posets $N C^{m}(W)$ are isomorphic to each other. If $m=1$, then $N C^{1}(W)$ can be identified with the set $N C(W)$ of non-crossing partitions for the (complex) reflection group $W$ as defined by Bessis and Corran (cf. [8] and [7, Sec. 13]; their definition extends the earlier definition by Bessis [6] and Brady and Watt [10] for real reflection groups).

The following result has been proved by a collaborative effort of several authors (see [7, Prop. 13.1]).

\footnotetext{
${ }^{2}$ An element of an irreducible well-generated complex reflection group $W$ of rank $n$ is called a Coxeter element if it is regular in the sense of Springer [33] (see also [24, Def. 11.21]) and of order $d_{n}$. An element of $W$ is called regular if it has an eigenvector which lies in no reflecting hyperplane of a reflection of $W$. It follows from an observation of Lehrer and Springer, proved uniformly by Lehrer and Michel [23] (see [24, Theorem 11.28]), that there is always a regular element of order $d_{n}$ in an irreducible well-generated complex reflection group $W$ of rank $n$. More generally, if a well-generated complex reflection group $W$ decomposes as $W \cong W_{1} \times W_{2} \times \cdots \times W_{k}$, where the $W_{i}$ 's are irreducible, then a Coxeter element of $W$ is an element of the form $c=c_{1} c_{2} \cdots c_{k}$, where $c_{i}$ is a Coxeter element of $W_{i}, i=1,2, \ldots, k$. If $W$ is a real reflection group, that is, if all generators in $T$ have order 2 , then the notion of generalised Coxeter element given above reduces to that of a Coxeter element in the classical sense (cf. [16, Sec. 3.16]).
} 
Theorem 1. Let $W$ be an irreducible well-generated complex reflection group, and let $d_{1} \leq d_{2} \leq \cdots \leq d_{n}$ be its degrees and $h:=d_{n}$ its Coxeter number. Then

$$
\left|N C^{m}(W)\right|=\prod_{i=1}^{n} \frac{m h+d_{i}}{d_{i}} .
$$

Remark 1. (1) The number in (2.2) is called the Fuß-Catalan number for the reflection group $W$.

(2) If $c$ is a Coxeter element of a well-generated complex reflection group $W$ of rank $n$, then $\ell_{T}(c)=n$. (This follows from [7, Sec. 7].)

We conclude this section by recalling the definition of decomposition numbers from [17, 18, 20. Although we need them here only for (very small) real reflection groups, and although, strictly speaking, they have been only defined for real reflection groups in [17, 18, 20], this definition can be extended to well-generated complex reflection groups without any extra effort, which we do now.

Given a well-generated complex reflection group $W$ of rank $n$, types $T_{1}, T_{2}, \ldots, T_{d}$ (in the sense of the classification of well-generated complex reflection groups) such that the sum of the ranks of the $T_{i}$ 's equals $n$, and a Coxeter element $c$, the decomposition number $N_{W}\left(T_{1}, T_{2}, \ldots, T_{d}\right)$ is defined as the number of "minimal" factorisations $c=c_{1} c_{2} \cdots c_{d}$, "minimal" meaning that $\ell_{T}\left(c_{1}\right)+\ell_{T}\left(c_{2}\right)+\cdots+\ell_{T}\left(c_{d}\right)=\ell_{T}(c)=n$, such that, for $i=1,2, \ldots, d$, the type of $c_{i}$ as a parabolic Coxeter element is $T_{i}$. (Here, the term "parabolic Coxeter element" means a Coxeter element in some parabolic subgroup. It follows from [31, Prop.6.3] that any element $c_{i}$ is indeed a Coxeter element in a unique parabolic subgroup of $W 3$ By definition, the type of $c_{i}$ is the type of this parabolic subgroup.) Since any two Coxeter elements are related to each other by conjugation plus field automorphism, the decomposition numbers are independent of the choice of the Coxeter element $c$.

The decomposition numbers for real reflection groups have been computed in [17, 18, 20]. To compute the decomposition numbers for well-generated complex reflection groups is a task that remains to be done.

\section{CyCliC Sieving I}

In this section we present the first cyclic sieving conjecture due to Armstrong [2, Conj. 5.4.7], and to Bessis and Reiner [9, Conj. 6.4].

Let $\phi: N C^{m}(W) \rightarrow N C^{m}(W)$ be the map defined by

$$
\left(w_{0} ; w_{1}, \ldots, w_{m}\right) \mapsto\left(\left(c w_{m} c^{-1}\right) w_{0}\left(c w_{m} c^{-1}\right)^{-1} ; c w_{m} c^{-1}, w_{1}, w_{2}, \ldots, w_{m-1}\right) .
$$

It is indeed not difficult to see that, if the $(m+1)$-tuple on the left-hand side is an element of $N C^{m}(W)$, then so is the $(m+1)$-tuple on the right-hand side. For $m=1$, this action reduces to conjugation by the Coxeter element $c$ (applied to $w_{1}$ ). Cyclic sieving arising from conjugation by $c$ has been the subject of [9].

\footnotetext{
${ }^{3}$ The uniqueness can be argued as follows: suppose that $c_{i}$ were a Coxeter element in two parabolic subgroups of $W$, say $U_{1}$ and $U_{2}$. Then it must also be a Coxeter element in the intersection $U_{1} \cap U_{2}$. On the other hand, the absolute length of a Coxeter element of a complex reflection group $U$ is always equal to $\operatorname{rk}(U)$, the rank of $U$. (This follows from the fact that, for each element $u$ of $U$, we have $\ell_{T}(u)=\operatorname{codim}(\operatorname{ker}(u-\mathrm{id}))$, with id denoting the identity element in $U$; see e.g. 31, Prop. 1.3]). We conclude that $\ell_{T}\left(c_{i}\right)=\operatorname{rk}\left(U_{1}\right)=\operatorname{rk}\left(U_{2}\right)=\operatorname{rk}\left(U_{1} \cap U_{2}\right)$, This implies that $U_{1}=U_{2}$.
} 
It is easy to see that $\phi^{m h}$ acts as the identity, where $h$ is the Coxeter number of $W$ (see (5.1) and Lemma 29 below). By slight abuse of notation, let $C_{1}$ be the cyclic group of order $m h$ generated by $\phi$. (The slight abuse consists in the fact that we insist on $C_{1}$ to be a cyclic group of order $m h$, while it may happen that the order of the action of $\phi$ given in (3.1) is actually a proper divisor of $m h$. )

Given these definitions, we are now in the position to state the first cyclic sieving conjecture of Armstrong, respectively of Bessis and Reiner. By the results of [19] and of this paper, it becomes the following theorem.

Theorem 2. For an irreducible well-generated complex reflection group $W$ and any $m \geq 1$, the triple $\left(N C^{m}(W), \mathrm{Cat}^{m}(W ; q), C_{1}\right)$, where $\mathrm{Cat}^{m}(W ; q)$ is the q-analogue of the Fuß-Catalan number defined by

$$
\operatorname{Cat}^{m}(W ; q):=\prod_{i=1}^{n} \frac{\left[m h+d_{i}\right]_{q}}{\left[d_{i}\right]_{q}},
$$

exhibits the cyclic sieving phenomenon in the sense of Reiner, Stanton and White [30]. Here, $n$ is the rank of $W, d_{1}, d_{2}, \ldots, d_{n}$ are the degrees of $W, h$ is the Coxeter number of $W$, and $[\alpha]_{q}:=\left(1-q^{\alpha}\right) /(1-q)$.

Remark 2. We write $\mathrm{Cat}^{m}(W)$ for $\mathrm{Cat}^{m}(W ; 1)$.

By definition of the cyclic sieving phenomenon, we have to prove that $\operatorname{Cat}^{m}(W ; q)$ is a polynomial in $q$ with non-negative integer coefficients, and that

$$
\left|\operatorname{Fix}_{N C^{m}(W)}\left(\phi^{p}\right)\right|=\left.\operatorname{Cat}^{m}(W ; q)\right|_{q=e^{2 \pi i p / m h}},
$$

for all $p$ in the range $0 \leq p<m h$. The first fact is established in the next section, while the proof of the second is achieved by making use of several auxiliary results, given in Section 5, to reduce the proof to a finite problem, and a subsequent case-by-case analysis. All details of this analysis can be found in [21, Sec. 6]. In the present paper, we content ourselves with discussing the cases where $W=G_{24}$ and where $W=G_{37}=E_{8}$, since these suffice to convey the flavour of the necessary computations.

\section{The $q$-Fusz-Catalan numbers $\operatorname{Cat}^{m}(W ; q)$}

The purpose of this section is to provide an elementary, self-contained proof of the fact that, for all irreducible complex reflection groups $W$, the $q$-Fuß-Catalan number $\mathrm{Cat}^{m}(W ; q)$ is a polynomial in $q$ with non-negative integer coefficients. For most of the groups, this is a known property. However, aside from the fact that, for many of the known cases, the proof is very indirect and uses deep algebraic results on rational Cherednik algebras, there still remained some cases where this property had not been formally established. The reader is referred to the "Theorem" in Section 1.6 of [15], which says that, under the assumption of a certain rank condition ([15, Hypothesis 2.4]), the $q$-Fuß-Catalan number $\operatorname{Cat}^{m}(W ; q)$ is a Hilbert series of a finite-dimensional quotient of the ring of invariants of $W$ and also the graded character of a finite-dimensional irreducible representation of a spherical rational Cherednik algebra associated with $W$. At present, this rank condition has been proven for all irreducible well-generated complex reflection groups apart from $G_{17}, G_{18}, G_{29}, G_{33}, G_{34}$; see [26, Tables 8 and 9 , column "rank"], and the recent paper [27], which establishes the result in the case of $G_{32}$. 
In the sequel, aside from the standard notation $[\alpha]_{q}=\left(1-q^{\alpha}\right) /(1-q)$ for $q$-integers, we shall also use the $q$-binomial coefficient, which is defined by

$$
\left[\begin{array}{l}
n \\
k
\end{array}\right]_{q}:= \begin{cases}1, & \text { if } k=0 \\
\frac{[n]_{q}[n-1]_{q} \cdots[n-k+1]_{q}}{[k]_{q}[k-1]_{q} \cdots[1]_{q}}, & \text { if } k>0 .\end{cases}
$$

We begin with several auxiliary results.

Proposition 3. For all non-negative integers $n$ and $k$, the q-binomial coefficient $\left[\begin{array}{l}n \\ k\end{array}\right]_{q}$ is a polynomial in $q$ with non-negative integer coefficients.

Proof. This is a well-known fact, which can be derived either from the recurrence relation(s) satisfied by the $q$-binomial coefficients (generalising Pascal's recurrence relation for binomial coefficients; cf. [1, eqs. (3.3.3) and (3.3.4)]), or from the fact that the $q$ binomial coefficient $\left[\begin{array}{l}n \\ k\end{array}\right]_{q}$ is the generating function for (integer) partitions with at most $k$ parts all of which are at most $n-k$ (cf. [1, Theorem 3.1]).

Proposition 4. For all non-negative integers $m$ and $n$, the q-Fuß-Catalan number of type $A_{n}$,

$$
\frac{1}{[(m+1) n+1]_{q}}\left[\begin{array}{c}
(m+1) n+1 \\
n
\end{array}\right]_{q},
$$

is a polynomial in $q$ with non-negative integer coefficients.

Proof. In [25, Sec. 3.3], Loehr proves that

$$
\begin{aligned}
& \frac{1}{[(m+1) n+1]_{q}}\left[\begin{array}{c}
(m+1) n+1 \\
n
\end{array}\right]_{q} \\
& =\sum_{v \in \mathcal{V}_{n}^{(m)}} q^{m\left(\begin{array}{c}
n \\
2
\end{array}\right)+\sum_{i \geq 0}\left(m\left(\begin{array}{c}
v_{i} \\
2
\end{array}\right)-i v_{i}\right)} \prod_{i \geq 1} q^{v_{i} \sum_{j=1}^{m}(m-j) v_{i-j}}\left[\begin{array}{c}
v_{i}+v_{i-1}+\cdots+v_{i-m}-1 \\
v_{i}
\end{array}\right]_{q},
\end{aligned}
$$

where $\mathcal{V}_{n}^{(m)}$ denotes the set of all sequences $v=\left(v_{0}, v_{1}, \ldots, v_{s}\right)$ (for some $s$ ) of nonnegative integers with $v_{0}>0, v_{s}>0$, and $v_{0}+v_{1}+\cdots+v_{s}=n$, and such that there is never a string of $m$ or more consecutive zeroes in $v$. By convention, $v_{i}=0$ for all negative $i$. His proof works by showing that the expressions on both sides of (4.1) satisfy the same recurrence relation and initial conditions, using classical $q$-binomial identities. We refer the reader to [25] for details. By Proposition 3, the expression on the right-hand side of (4.1) is manifestly a polynomial in $q$ with non-negative integer coefficients.

Lemma 5. If $a$ and $b$ are coprime positive integers, then

$$
\frac{[a b]_{q}}{[a]_{q}[b]_{q}}
$$

is a polynomial in $q$ of degree $(a-1)(b-1)$, all of whose coefficients are in $\{0,1,-1\}$. Moreover, if one disregards the coefficients which are 0 , then +1 's and (-1)'s alternate, and the constant coefficient as well as the leading coefficient of the polynomial equal +1 .

Proof. Let $\Phi_{n}(q)$ denote the $n$-th cyclotomic polynomial in $q$. Using the classical formula

$$
1-q^{n}=\prod_{d \mid n} \Phi_{d}(q)
$$


we see that

$$
\frac{(1-q)\left(1-q^{a b}\right)}{\left(1-q^{a}\right)\left(1-q^{b}\right)}=\prod_{\substack{d_{1}\left|a, d_{1} \neq 1 \\ d_{2}\right| a, d_{2} \neq 1}} \Phi_{d_{1} d_{2}}(q)
$$

so that, manifestly, the expression in (4.2) is a polynomial in $q$. The claim concerning the degree of this polynomial is obvious.

In order to establish the claim on the coefficients, we start with a sub-expression of (4.2),

$$
\frac{\left(1-q^{a b}\right)}{\left(1-q^{a}\right)\left(1-q^{b}\right)}=\left(\sum_{i=0}^{b-1} q^{i a}\right)\left(\sum_{j=0}^{\infty} q^{j b}\right)=\sum_{k=0}^{\infty} C_{k} q^{k},
$$

say. The assumption that $a$ and $b$ are coprime implies that $0 \leq C_{k} \leq 1$ for $k \leq$ $(a-1)(b-1)$. Multiplying both sides of (4.3) by $1-q$, we obtain the equation

$$
\frac{[a b]_{q}}{[a]_{q}[b]_{q}}=(1-q) \sum_{k=0}^{(a-1)(b-1)} C_{k} q^{k}+(1-q) \sum_{k=(a-1)(b-1)+1}^{\infty} C_{k} q^{k} .
$$

By our previous observation on the coefficients $C_{k}$ with $k \leq(a-1)(b-1)$, it is obvious that the coefficients of the first expression on the right-hand side of (4.4) are alternately +1 and -1 , when 0 's are disregarded. Since we already know that the left-hand side is a polynomial in $q$ of degree $(a-1)(b-1)$, we may ignore the second expression.

The proof is concluded by observing that the claims on the constant and leading coefficients are obvious.

Corollary 6. Let $a$ and $b$ be coprime positive integers, and let $\gamma$ be an integer with $\gamma \geq(a-1)(b-1)$. Then the expression

$$
\frac{[\gamma]_{q}[a b]_{q}}{[a]_{q}[b]_{q}}
$$

is a polynomial in $q$ with non-negative integer coefficients.

Proof. Let

$$
\frac{[a b]_{q}}{[a]_{q}[b]_{q}}=\sum_{k=0}^{(a-1)(b-1)} D_{k} q^{k}
$$

We then have

$$
\frac{[\gamma]_{q}[a b]_{q}}{[a]_{q}[b]_{q}}=\sum_{N=0}^{(a-1)(b-1)+\gamma-1} q^{N} \sum_{k=\max \{0, N-\gamma+1\}}^{N} D_{k} .
$$

If $N \leq \gamma-1$, then, by Lemma 5, the sum over $k$ on the right-hand side of (4.5) equals $1-1+1-1+\cdots$, which is manifestly non-negative. On the other hand, if $N>\gamma-1$, then we may rewrite the sum over $k$ on the right-hand side of (4.5) as

$$
\sum_{k=\max \{0, N-\gamma+1\}}^{N} D_{k}=\sum_{k=N-\gamma+1}^{(a-1)(b-1)} D_{k}=\sum_{k=0}^{(a-1)(b-1)+\gamma-1-N} D_{(a-1)(b-1)-k}
$$

Again, by Lemma 5, this sum equals $1-1+1-1+\cdots$, which is manifestly nonnegative. 
The next lemmas all have a very similar flavour, and so do their proofs. In order to avoid repetition, proof details are only provided for Lemmas 7 and 16, the proofs of Lemmas 91 15, 22 24 follow the pattern exhibited in the proof of Lemma 7, while the proofs of Lemmas 17 21 follow that of the proof of Lemma 15. Full details are found in [21, Sec. 4].

Lemma 7. Let $\alpha$ and $\beta$ be positive integers with $\alpha \geq 6$ and $\beta \geq 8$. Then the expression

$$
[\alpha]_{q^{3}}[\beta]_{q^{4}} \frac{[72]_{q}[3]_{q}[4]_{q}}{[8]_{q}[9]_{q}[12]_{q}}
$$

is a polynomial in $q$ with non-negative integer coefficients.

Proof. We have

$$
\begin{aligned}
& \frac{[72]_{q}[3]_{q}[4]_{q}}{[8]_{q}[9]_{q}[12]_{q}} \\
& \quad=\left(1-q^{3}+q^{9}-q^{15}+q^{18}\right)\left(1-q^{4}+q^{8}-q^{12}+q^{16}-q^{20}+q^{24}-q^{28}+q^{32}\right) .
\end{aligned}
$$

It should be observed that both factors on the right-hand side have the property that coefficients are in $\{0,1,-1\}$ and that $(+1)$ 's and $(-1)$ 's alternate, if one disregards the coefficients which are 0 . If we now apply the same idea as in the proof of Corollary 6 , then we see that $[\alpha]_{q^{3}}$ times the first factor is a polynomial in $q$ with non-negative integer coefficients, as is $[\beta]_{q^{4}}$ times the second factor. Taken together, this establishes the claim.

Lemma 8. Let $\alpha$ and $\beta$ be positive integers with $\alpha \geq 26$ and $\beta \geq 8$. Then the expression

$$
[\alpha]_{q}[\beta]_{q^{4}} \frac{[15]_{q}}{[3]_{q}[5]_{q}} \frac{[72]_{q}[3]_{q}[4]_{q}}{[8]_{q}[9]_{q}[12]_{q}}
$$

is a polynomial in $q$ with non-negative integer coefficients.

Lemma 9. Let $\alpha$ and $\beta$ be positive integers with $\alpha \geq 18$ and $\beta \geq 3$. Then the expression

$$
[\alpha]_{q^{3}}[\beta]_{q^{4}} \frac{[90]_{q}[3]_{q}[4]_{q}}{[5]_{q}[6]_{q}[9]_{q}}
$$

is a polynomial in $q$ with non-negative integer coefficients.

Lemma 10. Let $\alpha$ and $\beta$ be positive integers with $\alpha \geq 20$ and $\beta \geq 18$. Then the expression

$$
[\alpha]_{q}[\beta]_{q^{3}} \frac{[90]_{q}[3]_{q}}{[5]_{q}[6]_{q}[9]_{q}}
$$

is a polynomial in $q$ with non-negative integer coefficients.

Lemma 11. Let $\alpha$ be a positive integer with $\alpha \geq 26$. Then the expression

$$
[\alpha]_{q} \frac{[15]_{q}}{[3]_{q}[5]_{q}} \frac{[12]_{q^{3}}}{[3]_{q^{3}}[4]_{q^{3}}}
$$

is a polynomial in $q$ with non-negative integer coefficients. 
Lemma 12. Let $\alpha$ be a positive integer with $\alpha \geq 14$. Then the expression

$$
[\alpha]_{q} \frac{[15]_{q}}{[3]_{q}[5]_{q}} \frac{[6]_{q^{3}}}{[2]_{q^{3}}[3]_{q^{3}}}
$$

is a polynomial in $q$ with non-negative integer coefficients.

Lemma 13. Let $\alpha$ and $\beta$ be positive integers with $\alpha \geq 30$ and $\beta \geq 20$. Then the expression

$$
[\alpha]_{q}[\beta]_{q^{2}} \frac{[84]_{q}[2]_{q}}{[4]_{q}[6]_{q}[7]_{q}}
$$

is a polynomial in $q$ with non-negative integer coefficients.

Lemma 14. Let $\alpha$ and $\beta$ be positive integers with $\alpha \geq 24$ and $\beta \geq 68$. Then the expression

$$
[\alpha]_{q}[\beta]_{q} \frac{[105]_{q}}{[3]_{q}[5]_{q}[7]_{q}}
$$

is a polynomial in $q$ with non-negative integer coefficients.

Lemma 15. Let $\alpha$ and $\beta$ be positive integers with $\alpha \geq 24$ and $\beta \geq 34$. Then the expression

$$
[\alpha]_{q}[\beta]_{q} \frac{[70]_{q}}{[2]_{q}[5]_{q}[7]_{q}}
$$

is a polynomial in $q$ with non-negative integer coefficients.

Lemma 16. Let $\alpha$ and $\beta$ be positive integers with $\alpha \geq 4$ and $\beta \geq 2$. Then the expression

$$
[\alpha]_{q^{2}}[\beta]_{q^{5}} \frac{[30]_{q}[2]_{q}[3]_{q}[5]_{q}}{[6]_{q}[10]_{q}[15]_{q}}
$$

is a polynomial in $q$ with non-negative integer coefficients.

Proof. We have

$$
\frac{[30]_{q}[2]_{q}[3]_{q}[5]_{q}}{[6]_{q}[10]_{q}[15]_{q}}=1+q-q^{3}-q^{4}-q^{5}+q^{7}+q^{8} .
$$

If we multiply this expression by $[\alpha]_{q^{2}}$, then, for $\alpha=4$ we obtain

$$
1+q+q^{2}-q^{5}-q^{9}+q^{12}+q^{13}+q^{14}
$$

for $\alpha=5$ we obtain

$$
1+q+q^{2}-q^{5}+q^{8}-q^{11}+q^{14}+q^{15}+q^{16}
$$

and, for $\alpha \geq 6$, we obtain

$$
1+q+q^{2}-q^{5}+q^{8}+q^{10}+p_{1}(q)+q^{2 \alpha-4}+q^{2 \alpha-2}-q^{2 \alpha+1}+q^{2 \alpha+4}+q^{2 \alpha+5}+q^{2 \alpha+6},
$$

where $p_{1}(q)$ is a polynomial in $q$ with non-negative coefficients of order at least 11 and degree at most $2 \alpha-5$. In all cases it is obvious that the product of the result and $[\beta]_{q^{5}}$, with $\beta \geq 2$, is a polynomial in $q$ with non-negative coefficients. 
Lemma 17. Let $\alpha$ and $\beta$ be positive integers with $\alpha \geq 14$ and $\beta \geq 2$. Then the expression

$$
[\alpha]_{q}[\beta]_{q^{5}} \frac{[14]_{q}}{[2]_{q}[7]_{q}} \frac{[30]_{q}[2]_{q}[3]_{q}[5]_{q}}{[6]_{q}[10]_{q}[15]_{q}}
$$

is a polynomial in $q$ with non-negative integer coefficients.

Lemma 18. Let $\alpha$ and $\beta$ be positive integers with $\alpha \geq 32$ and $\beta \geq 12$. Then the expression

$$
[\alpha]_{q}[\beta]_{q^{2}} \frac{[35]_{q}}{[5]_{q}[7]_{q}} \frac{[30]_{q}[2]_{q}[3]_{q}[5]_{q}}{[6]_{q}[10]_{q}[15]_{q}}
$$

is a polynomial in $q$ with non-negative integer coefficients.

Lemma 19. Let $\alpha$ and $\beta$ be positive integers with $\alpha \geq 16$ and $\beta \geq 2$. Then the expression

$$
[\alpha]_{q^{2}}[\beta]_{q^{5}} \frac{[60]_{q}[2]_{q}[3]_{q}[5]_{q}}{[10]_{q}[12]_{q}[15]_{q}}
$$

is a polynomial in $q$ with non-negative integer coefficients.

Lemma 20. Let $\alpha$ and $\beta$ be positive integers with $\alpha \geq 56$ and $\beta \geq 4$. Then the expression

$$
[\alpha]_{q}[\beta]_{q^{2}} \frac{[35]_{q}}{[5]_{q}[7]_{q}} \frac{[60]_{q}[2]_{q}[3]_{q}[5]_{q}}{[10]_{q}[12]_{q}[15]_{q}}
$$

is a polynomial in $q$ with non-negative integer coefficients.

Lemma 21. Let $\alpha$ and $\beta$ be positive integers with $\alpha \geq 38$ and $\beta \geq 2$. Then the expression

$$
[\alpha]_{q}[\beta]_{q^{5}} \frac{[14]_{q}}{[2]_{q}[7]_{q}} \frac{[60]_{q}[2]_{q}[3]_{q}[5]_{q}}{[10]_{q}[12]_{q}[15]_{q}}
$$

is a polynomial in $q$ with non-negative integer coefficients.

Lemma 22. Let $\alpha$ and $\beta$ be positive integers with $\alpha \geq 30$ and $\beta \geq 26$. Then the expression

$$
[\alpha]_{q}[\beta]_{q^{3}} \frac{[126]_{q}[3]_{q}}{[6]_{q}[7]_{q}[9]_{q}}
$$

is a polynomial in $q$ with non-negative integer coefficients.

Lemma 23. Let $\alpha$ and $\beta$ be positive integers with $\alpha \geq 66$ and $\beta \geq 54$. Then the expression

$$
[\alpha]_{q}[\beta]_{q^{3}} \frac{[252]_{q}[3]_{q}}{[7]_{q}[9]_{q}[12]_{q}}
$$

is a polynomial in $q$ with non-negative integer coefficients.

Lemma 24. Let $\alpha$ and $\beta$ be positive integers with $\alpha \geq 54$ and $\beta \geq 34$. Then the expression

$$
[\alpha]_{q}[\beta]_{q^{2}} \frac{[140]_{q}[2]_{q}}{[4]_{q}[7]_{q}[10]_{q}}
$$

is a polynomial in $q$ with non-negative integer coefficients. 
We are now ready for the proof of the main result of this section.

Theorem 25. For all irreducible well-generated complex reflection groups and positive integers $m$, the $q$-Fuß-Catalan number $\mathrm{Cat}^{m}(W ; q)$ is a polynomial in $q$ with nonnegative integer coefficients.

Proof. First, let $W=A_{n}$. In this case, the degrees are $2,3, \ldots, n+1$, and hence

$$
\operatorname{Cat}^{m}\left(A_{n} ; q\right)=\frac{1}{[(m+1) n+1]_{q}}\left[\begin{array}{c}
(m+1) n+1 \\
n
\end{array}\right]_{q},
$$

which, by Proposition 4, is a polynomial in $q$ with non-negative integer coefficients.

Next, let $W=G(d, 1, n)$. In this case, the degrees are $d, 2 d, \ldots, n d$, and hence

$$
\operatorname{Cat}^{m}(G(d, 1, n) ; q)=\left[\begin{array}{c}
(m+1) n \\
n
\end{array}\right]_{q^{d}},
$$

which, by Proposition 3, is a polynomial in $q$ with non-negative integer coefficients.

Now, let $W=G(e, e, n)$. In this case, the degrees are $e, 2 e, \ldots,(n-1) e, n$, and hence

$$
\begin{aligned}
\operatorname{Cat}^{m}(G(e, e, n) ; q) & =\frac{[m(n-1) e+n]_{q}}{[n]_{q}} \prod_{i=1}^{n-1} \frac{[m(n-1) e+i e]_{q}}{[i e]_{q}} \\
& =\left[\begin{array}{c}
(m+1)(n-1) \\
n-1
\end{array}\right]_{q^{e}}+q^{n}[e]_{q^{n}}\left[\begin{array}{c}
(m+1)(n-1) \\
n
\end{array}\right]_{q^{e}},
\end{aligned}
$$

which, by Proposition 3, is a polynomial in $q$ with non-negative integer coefficients.

It remains to verify the claim for the exceptional groups.

For the groups $W=G_{6}, G_{9}, G_{14}, G_{17}, G_{21}$, and partially for the groups $W=G_{20}, G_{23}$, $G_{28}, G_{30}, G_{33}, G_{35}, G_{36}, G_{37}$ (depending on congruence properties of the parameter $m$ ), polynomiality and non-negativity of coefficients of the corresponding $q$-Fuß-Catalan number can be directly read off by a proper rearrangement of the terms in the defining expression; for example, for $W=G_{21}$ (with degrees given by 12,60 ) we have

$$
\operatorname{Cat}^{m}\left(G_{21} ; q\right)=\frac{[60 m+12]_{q}[60 m+60]_{q}}{[12]_{q}[60]_{q}}=[5 m+1]_{q^{12}}[m+1]_{q^{60}},
$$

which is manifestly a polynomial in $q$ with non-negative integer coefficients.

For the groups $G_{5}, G_{10}, G_{18}, G_{26}, G_{27}, G_{29}, G_{34}$, the terms in the defining expression of the corresponding $q$-Fuß-Catalan number can be arranged in a manner so that a $q$-binomial coefficient appears; polynomiality and non-negativity of coefficients then follow from Proposition 3. For example, for $W=G_{34}$ (with degrees given by $6,12,18,24,30,42)$ we have

$$
\begin{aligned}
\operatorname{Cat}^{m}\left(G_{34} ; q\right) & =\frac{[42 m+6]_{q}[42 m+12]_{q}[42 m+18]_{q}[42 m+24]_{q}[42 m+30]_{q}[42 m+42]_{q}}{[6]_{q}[12]_{q}[18]_{q}[24]_{q}[30]_{q}[42]_{q}} \\
& =[m+1]_{q^{42}}\left[\begin{array}{c}
7 m+5 \\
5
\end{array}\right]_{q^{6}},
\end{aligned}
$$

which, written in this form, is obviously a polynomial in $q$ with non-negative integer coefficients. 
On the other hand, for the groups $G_{4}, G_{8}, G_{16}, G_{25}, G_{32}$, the terms in the defining expression of the corresponding $q$-Fuß-Catalan number can be arranged in a manner so that a $q$-Fuß-Catalan number of type $A$ appears and Proposition 4 applies; for example, for $W=G_{32}$ (with degrees given by $12,18,24,30$ ) we have

$$
\begin{aligned}
\operatorname{Cat}^{m}\left(G_{32} ; q\right) & =\frac{[30 m+12]_{q}[30 m+18]_{q}[30 m+24]_{q}[30 m+30]_{q}}{[12]_{q}[18]_{q}[24]_{q}[30]_{q}} \\
& =\frac{1}{[5 m+6]_{q^{6}}}\left[\begin{array}{c}
5 m+6 \\
5
\end{array}\right]_{q^{6}},
\end{aligned}
$$

which indeed fits into the framework of Proposition 4 and, hence, is a polynomial in $q$ with non-negative integer coefficients.

In the other cases, the more "specialised" auxiliary results given in Corollary 6 and Lemmas 724 have to be applied. For the sake of illustration, we exhibit one example for each of them below, with full details being provided in [21, Sec. 4]. In general, the idea is that, given a rational expression consisting of cyclotomic factors, as in the definition of the $q$-Fuß-Catalan numbers, one tries to place denominator factors below appropriate numerator factors so that one can divide out the denominator factor completely. For example, if we were to encounter the expression

$$
\frac{[30 m+12]_{q} \cdot(\text { other terms })}{[12]_{q} \cdot(\text { other terms })}
$$

and know that $m$ is even, then we would try to simplify this to

$$
\left[\frac{5 m+2}{2}\right]_{q^{12}} \cdot \frac{(\text { other terms })}{\text { (other terms) }}
$$

where $\left[\frac{5 m+2}{2}\right]_{q^{12}}$ is manifestly a polynomial in $q$ with non-negative integer coefficients. On the other hand, in a situation where two denominator factors "want" to divide a single numerator factor, we "extract" as much as we can from the numerator factor and compensate by additional "fudge" factors. To be more concrete, if we encounter the expression

$$
\frac{[14 m+14]_{q} \cdot(\text { other terms })}{[6]_{q}[14]_{q} \cdot(\text { other terms })}
$$

and we know that $m \equiv 0(\bmod 3)$, then we would try the rewriting

$$
\left[\frac{m+1}{3}\right]_{q^{42}} \frac{[21]_{q^{2}}}{[3]_{q^{2}}[7]_{q^{2}}[2]_{q}} \cdot \frac{\text { (other terms) }}{\text { (other terms) }}
$$

with the idea that we might find somewhere else a term $[2 \alpha]_{q}$, which could be combined with the term $[2]_{q}$ in the denominator into $[2 \alpha]_{q} /[2]_{q}=[\alpha]_{q^{2}}$, and then apply Corollary 6 to see that

$$
[\alpha]_{q^{2}} \frac{[21]_{q^{2}}}{[3]_{q^{2}}[7]_{q^{2}}}
$$

is a polynomial in $q$ with non-negative integer coefficients (provided $\alpha$ is at least 12), with $\left[\frac{m+1}{3}\right]_{q^{42}}$ being such a polynomial in any case.

In situations where three denominator factors "want" to divide a single numerator factor, one has to perform more complicated rearrangements, in order to be able to apply one of the Lemmas 724 . 
For example, for $W=G_{24}$, the degrees are 4,6,14, and hence

$$
\mathrm{Cat}^{m}\left(G_{24} ; q\right)=\frac{[14 m+4]_{q}[14 m+6]_{q}[14 m+14]_{q}}{[4]_{q}[6]_{q}[14]_{q}}
$$

We have

$$
\operatorname{Cat}^{m}\left(G_{24} ; q\right)= \begin{cases}{\left[\frac{7 m}{2}+1\right]_{q^{4}}\left[\frac{14 m}{6}+1\right]_{q^{6}}[m+1]_{q^{14}},} & \text { if } m \equiv 0(\bmod 6), \\ {\left[\frac{7 m+2}{3}\right]_{q^{6}}\left[\frac{7 m+3}{2}\right]_{q^{4}}[m+1]_{q^{14}},} & \text { if } m \equiv 1(\bmod 6), \\ {\left[\frac{7 m}{2}+1\right]_{q^{4}}[7 m+3]_{q^{2}}\left[\frac{m+1}{3}\right]_{q^{42}} \frac{[21]_{q^{2}}}{[3]_{q_{2}}[7]_{q^{2}}},} & \text { if } m \equiv 2(\bmod 6), \\ {[7 m+2]_{q^{2}}\left[\frac{7 m}{3}+1\right]_{q^{6}}\left[\frac{m+1}{2}\right]_{q^{28}} \frac{[14]_{q^{2}}}{[2]_{q^{2}}[7]_{q^{2}}},} & \text { if } m \equiv 3(\bmod 6), \\ {\left[\frac{7 m+2}{6}\right]_{q^{12}} \frac{[6]_{q^{2}}}{[2]_{q^{2}}[3]_{q^{2}}}[7 m+3]_{q^{2}}[m+1]_{q^{14}},} & \text { if } m \equiv 4(\bmod 6), \\ {[7 m+2]_{q^{2}}\left[\frac{7 m+3}{2}\right]_{q^{4}}\left[\frac{m+1}{3}\right]_{q^{42}} \frac{[21]_{q^{2}}}{[3]_{q^{2}}[7]_{q^{2}}},} & \text { if } m \equiv 5(\bmod 6),\end{cases}
$$

which, by Corollary 6, are polynomials in $q$ with non-negative integer coefficients in all cases.

For $W=G_{30}=H_{4}$, the degrees are 2, 12, 20,30, and hence

$$
\operatorname{Cat}^{m}\left(H_{4} ; q\right)=\frac{[30 m+2]_{q}[30 m+12]_{q}[30 m+20]_{q}[30 m+30]_{q}}{[2]_{q}[12]_{q}[20]_{q}[30]_{q}} .
$$

If $m$ is odd, then we may write

$$
\operatorname{Cat}^{m}\left(H_{4} ; q\right)=\left[\frac{15 m+1}{2}\right]_{q^{4}}[5 m+2]_{q^{6}}[3 m+2]_{q^{10}}\left[\frac{m+1}{2}\right]_{q^{60}} \frac{[30]_{q^{2}}[2]_{q^{2}}[3]_{q^{2}}[5]_{q^{2}}}{[6]_{q^{6}}[10]_{q^{2}}[15]_{q^{2}}}
$$

which, by Lemma 16, is a polynomial in $q$ with non-negative integer coefficients.

For $W=G_{35}=E_{6}$, the degrees are $2,5,6,8,9,12$, and hence

$$
\operatorname{Cat}^{m}\left(E_{6} ; q\right)=\frac{[12 m+2]_{q}[12 m+5]_{q}[12 m+6]_{q}[12 m+8]_{q}[12 m+9]_{q}[12 m+12]_{q}}{[2]_{q}[5]_{q}[6]_{q}[8]_{q}[9]_{q}[12]_{q}} .
$$

If $m \equiv 5(\bmod 30)$, then we have

$$
\begin{aligned}
\operatorname{Cat}^{m}\left(E_{6} ; q\right)=[6 m+1]_{q^{2}}\left[\frac{12 m+5}{5}\right]_{q^{5}}[2 m & +1]_{q^{6}} \\
& \times[3 m+2]_{q^{4}}[4 m+3]_{q^{3}}\left[\frac{m+1}{6}\right]_{q^{72}} \frac{[72]_{q}[3]_{q}[4]_{q}}{[8]_{q}[9]_{q}[12]_{q}},
\end{aligned}
$$

which, by Lemma 7, is a polynomial in $q$ with non-negative integer coefficients.

If $m \equiv 7(\bmod 30)$, then we have

$$
\begin{aligned}
\operatorname{Cat}^{m}\left(E_{6} ; q\right)=\left[\frac{6 m+1}{2}\right]_{q^{4}}[12 m & +5]_{q}\left[\frac{2 m+1}{15}\right]_{q^{90}} \\
& \times \frac{[90]_{q}[3]_{q}[4]_{q}}{[5]_{q}[6]_{q}[9]_{q}}[3 m+2]_{q^{4}}[4 m+3]_{q^{3}}\left[\frac{m+1}{2}\right]_{q^{24}} \frac{[6]_{q^{4}}}{[2]_{q^{4}}[3]_{q^{4}}},
\end{aligned}
$$

which, by Corollary [6 and Lemma 9, is a polynomial in $q$ with non-negative integer coefficients. 
If $m \equiv 8(\bmod 30)$, then we have

$$
\begin{aligned}
\operatorname{Cat}^{m}\left(E_{6} ; q\right)=[6 m+1]_{q^{2}}[12 m+5]_{q}[2 m+1]_{q^{6}} & {\left[\frac{3 m+2}{2}\right]_{q^{8}} } \\
& \times\left[\frac{4 m+3}{5}\right]_{q^{15}} \frac{[15]_{q}}{[3]_{q}[5]_{q}}\left[\frac{m+1}{3}\right]_{q^{36}} \frac{[12]_{q^{3}}}{[3]_{q^{3}}[4]_{q^{3}}},
\end{aligned}
$$

which, by Lemma 11, is a polynomial in $q$ with non-negative integer coefficients.

If $m \equiv 13(\bmod 30)$, then we have

$$
\begin{aligned}
\operatorname{Cat}^{m}\left(E_{6} ; q\right)=[6 m+1]_{q^{2}}[12 m+5]_{q}\left[\frac{2 m+1}{3}\right]_{q^{18}} \frac{[6]_{q^{3}}}{[2]_{q^{3}}[3]_{q^{3}}} \\
\times[3 m+2]_{q^{4}}\left[\frac{4 m+3}{5}\right]_{q^{15}} \frac{[15]_{q}}{[3]_{q}[5]_{q}}\left[\frac{m+1}{2}\right]_{q^{24}} \frac{[6]_{q^{4}}}{[2]_{q^{4}}[3]_{q^{4}}}
\end{aligned}
$$

which, by Lemma 12, is a polynomial in $q$ with non-negative integer coefficients.

If $m \equiv 22(\bmod 30)$, then we have

$$
\begin{aligned}
\operatorname{Cat}^{m}\left(E_{6} ; q\right)=[6 m+1]_{q^{2}}[12 m+5]_{q}\left[\frac{2 m+1}{15}\right]_{q^{90}} \frac{[90]_{q}[3]_{q}}{[5]_{q}[6]_{q}[9]_{q}} \\
\times\left[\frac{3 m+2}{2}\right]_{q^{8}}[4 m+3]_{q^{3}}[m+1]_{q^{12}},
\end{aligned}
$$

which, by Lemma 10, is a polynomial in $q$ with non-negative integer coefficients.

If $m \equiv 23(\bmod 30)$, then we have

$$
\begin{aligned}
\operatorname{Cat}^{m}\left(E_{6} ; q\right)=[6 m+1]_{q^{2}}[12 m+ & 5]_{q}[2 m+1]_{q^{6}} \\
& \times[3 m+2]_{q^{4}}\left[\frac{4 m+3}{5}\right]_{q^{15}} \frac{[15]_{q}}{[3]_{q}[5]_{q}}\left[\frac{m+1}{6}\right]_{q^{72}} \frac{[72]_{q}[3]_{q}[4]_{q}}{[8]_{q}[9]_{q}[12]_{q}},
\end{aligned}
$$

which, by Lemma 8 , is a polynomial in $q$ with non-negative integer coefficients.

For $W=G_{36}=E_{7}$, the degrees are $2,6,8,10,12,14,18$, and hence

$$
\begin{aligned}
\operatorname{Cat}^{m}\left(E_{7} ; q\right)=\frac{[18 m+2]_{q}[18 m+6]_{q}[18 m+8]_{q}[18 m+10]_{q}}{[2]_{q}[6]_{q}[8]_{q}[10]_{q}} \\
\times \frac{[18 m+12]_{q}[18 m+14]_{q}[18 m+18]_{q}}{[12]_{q}[14]_{q}[18]_{q}} .
\end{aligned}
$$

If $m \equiv 18(\bmod 140)$, then we have

$$
\begin{aligned}
\operatorname{Cat}^{m}\left(E_{7} ; q\right)=[9 m & +1]_{q^{2}}\left[\frac{3 m+1}{5}\right]_{q^{30}} \frac{[15]_{q^{2}}}{[3]_{q^{2}}[5]_{q^{2}}} \\
& \times\left[\frac{9 m+4}{2}\right]_{q^{4}}[9 m+5]_{q^{2}}\left[\frac{3 m+2}{28}\right]_{q^{168}} \frac{[84]_{q^{2}}[2]_{q^{2}}}{[4]_{q^{2}}[6]_{q^{2}}[7]_{q^{2}}}[9 m+7]_{q^{2}}[m+1]_{q^{18}}
\end{aligned}
$$

which, by Corollary 6 and Lemma 13, is a polynomial in $q$ with non-negative integer coefficients. 
If $m \equiv 23(\bmod 140)$, then we have

$$
\begin{aligned}
\operatorname{Cat}^{m}\left(E_{7} ; q\right)=\left[\frac{9 m+1}{4}\right]_{q^{8}}\left[\frac{3 m+1}{35}\right]_{q^{210}} \frac{[105]_{q^{2}}}{[3]_{q^{2}}[5]_{q^{2}}[7]_{q^{2}}}[9 m+4]_{q^{2}}[9 m+5]_{q^{2}} \\
\times[3 m+2]_{q^{6}}[9 m+7]_{q^{2}}\left[\frac{m+1}{2}\right]_{q^{36}} \frac{[6]_{q^{6}}}{[2]_{q^{6}}[3]_{q^{6}}},
\end{aligned}
$$

which, by Corollary 6 and Lemma 14, is a polynomial in $q$ with non-negative integer coefficients.

If $m \equiv 54(\bmod 140)$, then we have

$$
\begin{aligned}
\operatorname{Cat}^{m}\left(E_{7} ; q\right)=[9 m+1]_{q^{2}}[3 m+1]_{q^{6}}\left[\frac{9 m+4}{70}\right]_{q^{140}} \frac{[70]_{q^{2}}}{[2]_{q^{2}}[5]_{q^{2}}[7]_{q^{2}}}[9 m+5]_{q^{2}} \\
\times\left[\frac{3 m+2}{4}\right]_{q^{24}} \frac{[6]_{q^{4}}}{[2]_{q^{4}}[3]_{q^{4}}}[9 m+7]_{q^{2}}[m+1]_{q^{18}} .
\end{aligned}
$$

If one decomposes $[9 m+7]_{q^{2}}$ as $\left[\frac{9 m}{2}+4\right]_{q^{4}}+q^{2}\left[\frac{9 m}{2}+3\right]_{q^{4}}$, then one sees that, by Corollary 6 and Lemma 15, this is a polynomial in $q$ with non-negative integer coefficients.

For $W=G_{37}=E_{8}$, the degrees are $2,8,12,14,18,20,24,30$, and hence

$$
\begin{aligned}
\left.\operatorname{Cat}^{m}\left(E_{7} ; q\right)=\frac{[30 m+2]_{q}[30 m}{}+8\right]_{q}[30 m+12]_{q}[30 m+14]_{q} \\
{[2]_{q}[8]_{q}[12]_{q}[14]_{q} } \\
\times \frac{[30 m+18]_{q}[30 m+20]_{q}[30 m+24]_{q}[30 m+30]_{q}}{[18]_{q}[20]_{q}[24]_{q}[30]_{q}} .
\end{aligned}
$$

If $m \equiv 3(\bmod 84)$, then we have

$$
\begin{aligned}
\operatorname{Cat}^{m}\left(E_{8} ; q\right)=\left[\frac{15 m+1}{2}\right]_{q^{4}}\left[\frac{15 m+4}{7}\right]_{q^{14}}[5 m+2]_{q^{6}}\left[\frac{15 m+7}{4}\right]_{q^{8}}\left[\frac{5 m+3}{6}\right]_{q^{36}} \frac{[6]_{q^{6}}}{[2]_{q^{6}}[3]_{q^{6}}} \\
\times[3 m+2]_{q^{10}}[5 m+4]_{q^{6}}\left[\frac{m+1}{4}\right]_{q^{120}} \frac{[60]_{q^{2}}[2]_{q^{2}}[3]_{q^{2}}[5]_{q^{2}}}{[10]_{q^{2}}[12]_{q^{2}}[15]_{q^{2}}}
\end{aligned}
$$

which, by Corollary 6 and Lemma 19, is a polynomial in $q$ with non-negative integer coefficients.

If $m \equiv 8(\bmod 84)$, then we have

$$
\begin{aligned}
\operatorname{Cat}^{m}\left(E_{8} ; q\right)=[15 m+1]_{q^{2}}\left[\frac{15 m+4}{4}\right]_{q^{8}}\left[\frac{5 m+2}{42}\right]_{q^{252}} \frac{[126]_{q^{2}}[3]_{q^{2}}}{[6]_{q^{2}}[7]_{q^{2}}[9]_{q^{2}}}[15 m+7]_{q^{2}}[5 m+3]_{q^{6}} \\
\times\left[\frac{3 m+2}{2}\right]_{q^{20}}\left[\frac{5 m+4}{4}\right]_{q^{24}}[m+1]_{q^{30}},
\end{aligned}
$$

which, by Lemma 22, is a polynomial in $q$ with non-negative integer coefficients.

If $m \equiv 11(\bmod 84)$, then we have

$$
\begin{aligned}
\operatorname{Cat}^{m}\left(E_{8} ; q\right)=\left[\frac{15 m+1}{2}\right]_{q^{4}} & {[15 m+4]_{q^{2}}\left[\frac{5 m+2}{3}\right]_{q^{18}}\left[\frac{15 m+7}{4}\right]_{q^{8}}\left[\frac{5 m+3}{2}\right]_{q^{12}} } \\
& \times\left[\frac{3 m+2}{7}\right]_{q^{70}} \frac{[35]_{q^{2}}}{[5]_{q^{2}}[7]_{q^{2}}}[5 m+4]_{q^{6}}\left[\frac{m+1}{4}\right]_{q^{120}} \frac{[60]_{q^{2}}[2]_{q^{2}}[3]_{q^{2}}[5]_{q^{2}}}{[10]_{q^{2}}[12]_{q^{2}}[15]_{q^{2}}}
\end{aligned}
$$


which, by Corollary 6 and Lemma 20, is a polynomial in $q$ with non-negative integer coefficients.

If $m \equiv 16(\bmod 84)$, then we have

$$
\begin{aligned}
\operatorname{Cat}^{m}\left(E_{8} ; q\right)=[15 m+1]_{q^{2}}\left[\frac{15 m+4}{4}\right]_{q^{8}}\left[\frac{5 m+2}{2}\right]_{q^{12}}[15 m+7]_{q^{2}}[5 m+3]_{q^{6}} \\
\times\left[\frac{3 m+2}{2}\right]_{q^{20}}\left[\frac{5 m+4}{84}\right]_{q^{504}} \frac{[252]_{q^{2}}[3]_{q^{2}}}{[7]_{q^{2}}[9]_{q^{2}}[12]_{q^{2}}}[m+1]_{q^{30}},
\end{aligned}
$$

which, by Lemma 23, is a polynomial in $q$ with non-negative integer coefficients.

If $m \equiv 18(\bmod 84)$, then we have

$$
\begin{aligned}
\operatorname{Cat}^{m}\left(E_{8} ; q\right)=[15 m+1]_{q^{2}}\left[\frac{15 m+4}{2}\right]_{q^{4}}\left[\frac{5 m+2}{4}\right]_{q^{24}}[15 m+7]_{q^{2}}\left[\frac{5 m+3}{3}\right]_{q^{18}} \\
\\
\quad\left[\frac{3 m+2}{28}\right]_{q^{280}} \frac{[140]_{q^{2}}[2]_{q^{2}}}{[4]_{q^{2}}[7]_{q^{2}}[10]_{q^{2}}}\left[\frac{5 m+4}{2}\right]_{q^{12}}[m+1]_{q^{30}},
\end{aligned}
$$

which, by Lemma 24, is a polynomial in $q$ with non-negative integer coefficients.

If $m \equiv 21(\bmod 84)$, then we have

$$
\begin{aligned}
\operatorname{Cat}^{m}\left(E_{8} ; q\right)=\left[\frac{15 m+1}{4}\right]_{q^{8}}[15 m+ & 4]_{q^{2}}[5 m+2]_{q^{6}}\left[\frac{15 m+7}{14}\right]_{q^{28}} \frac{[14]_{q^{2}}}{[2]_{q^{2}}[7]_{q^{2}}}\left[\frac{5 m+3}{12}\right]_{q^{72}} \frac{[12]_{q^{6}}}{[3]_{q^{6}}[4]_{q^{6}}} \\
& \times[3 m+2]_{q^{10}}[5 m+4]_{q^{6}}\left[\frac{m+1}{2}\right]_{q^{60}} \frac{[30]_{q^{2}}[2]_{q^{2}}[3]_{q^{2}}[5]_{q^{2}}}{[6]_{q^{2}}[10]_{q^{2}}[15]_{q^{2}}}
\end{aligned}
$$

which, by Corollary [6 and Lemma 17, is a polynomial in $q$ with non-negative integer coefficients.

If $m \equiv 25(\bmod 84)$, then we have

$$
\begin{aligned}
& \operatorname{Cat}^{m}\left(E_{8} ; q\right)=\left[\frac{15 m+1}{4}\right]_{q^{8}} {[15 m+4]_{q^{2}}[5 m+2]_{q^{6}}\left[\frac{15 m+7}{2}\right]_{q^{4}}\left[\frac{5 m+3}{4}\right]_{q^{24}} } \\
& \times\left[\frac{3 m+2}{7}\right]_{q^{70}} \frac{[35]_{q^{2}}}{[5]_{q^{2}}[7]_{q^{2}}}\left[\frac{5 m+4}{3}\right]_{q^{18}}\left[\frac{m+1}{2}\right]_{q^{60}} \frac{[30]_{q^{2}}[2]_{q^{2}}[3]_{q^{2}}[5]_{q^{2}}}{[6]_{q^{2}}[10]_{q^{2}}[15]_{q^{2}}}
\end{aligned}
$$

which, by Lemma 18, is a polynomial in $q$ with non-negative integer coefficients.

If $m \equiv 27(\bmod 84)$, then we have

$$
\begin{aligned}
\operatorname{Cat}^{m}\left(E_{8} ; q\right)=\left[\frac{15 m+1}{14}\right]_{q^{28}} \frac{[14]_{q^{2}}}{[2]_{q^{2}}[7]_{q^{2}}}[15 m+4]_{q^{2}}[5 m+2]_{q^{6}}\left[\frac{15 m+7}{4}\right]_{q^{8}}\left[\frac{5 m+3}{6}\right]_{q^{36}} \frac{[6]_{q^{6}}}{[2]_{q^{6}}[3]_{q^{6}}} \\
\times[3 m+2]_{q^{10}}[5 m+4]_{q^{6}}\left[\frac{m+1}{4}\right]_{q^{120}} \frac{[60]_{q^{2}}[2]_{q^{2}}[3]_{q^{2}}[5]_{q^{2}}}{[10]_{q^{2}}[12]_{q^{2}}[15]_{q^{2}}}
\end{aligned}
$$

which, by Corollary 6 and Lemma 21, is a polynomial in $q$ with non-negative integer coefficients.

All other cases are disposed of in a similar fashion.

\section{Auxiliary Results I}

This section collects several auxiliary results which allow us to reduce the problem of proving Theorem 2, or the equivalent statement (3.3), for the 26 exceptional groups listed in Section 2 to a finite problem. While Lemmas 27 and 28 cover special choices of the parameters, Lemmas 26 and 30 afford an inductive procedure. More precisely, 
if we assume that we have already verified Theorem 2 for all groups of smaller rank, then Lemmas 26 and 30, together with Lemmas 27 and 31, reduce the verification of Theorem 2 for the group that we are currently considering to a finite problem; see Remark 3. The final lemma of this section, Lemma 32, disposes of complex reflection groups with a special property satisfied by their degrees.

Let $p=a m+b, 0 \leq b<m$. We have

$$
\begin{array}{r}
\phi^{p}\left(\left(w_{0} ; w_{1}, \ldots, w_{m}\right)\right) \\
=\left(* ; c^{a+1} w_{m-b+1} c^{-a-1}, c^{a+1} w_{m-b+2} c^{-a-1}, \ldots, c^{a+1} w_{m} c^{-a-1},\right. \\
\left.c^{a} w_{1} c^{-a}, \ldots, c^{a} w_{m-b} c^{-a}\right),
\end{array}
$$

where $*$ stands for the element of $W$ which is needed to complete the product of the components to $c$.

Lemma 26. It suffices to check (3.3) for $p$ a divisor of $m$. More precisely, let $p$ be a divisor of $m h$, and let $k$ be another positive integer with $\operatorname{gcd}(k, m h / p)=1$, then we have

$$
\left.\operatorname{Cat}^{m}(W ; q)\right|_{q=e^{2 \pi i p / m h}}=\left.\operatorname{Cat}^{m}(W ; q)\right|_{q=e^{2 \pi i k p / m h}}
$$

and

$$
\left|\operatorname{Fix}_{N C^{m}(W)}\left(\phi^{p}\right)\right|=\left|\operatorname{Fix}_{N C^{m}(W)}\left(\phi^{k p}\right)\right| .
$$

Proof. For (5.2), this follows immediately from

$$
\lim _{q \rightarrow \zeta} \frac{[\alpha]_{q}}{[\beta]_{q}}= \begin{cases}\frac{\alpha}{\beta} & \text { if } \alpha \equiv \beta \equiv 0 \quad(\bmod d) \\ 1 & \text { otherwise }\end{cases}
$$

where $\zeta$ is a $d$-th root of unity and $\alpha, \beta$ are non-negative integers such that $\alpha \equiv \beta$ $(\bmod d)$.

In order to establish (5.3), suppose that $x \in \operatorname{Fix}_{N C^{m}(W)}\left(\phi^{p}\right)$, that is, $x \in N C^{m}(W)$ and $\phi^{p}(x)=x$. It obviously follows that $\phi^{k p}(x)=x$, so that $x \in \operatorname{Fix}_{N C^{m}(W)}\left(\phi^{k p}\right)$. To establish the converse, note that, if $\operatorname{gcd}(k, m h / p)=1$, then there exists $k^{\prime}$ with $k^{\prime} k \equiv 1\left(\bmod \frac{m h}{p}\right)$. It follows that, if $x \in \operatorname{Fix}_{N C^{m}(W)}\left(\phi^{k p}\right)$, that is, if $x \in N C^{m}(W)$ and $\phi^{k p}(x)=x$, then $x=\phi^{k^{\prime} k p}(x)=\phi^{p}(x)$, whence $x \in \operatorname{Fix}_{N C^{m}(W)}\left(\phi^{p}\right)$.

Lemma 27. Let $p$ be a divisor of $m$. If $p$ is divisible by $m$, then (3.3) is true.

Proof. According to (5.1), the action of $\phi^{p}$ on $N C^{m}(W)$ is described by

$$
\phi^{p}\left(\left(w_{0} ; w_{1}, \ldots, w_{m}\right)\right)=\left(* ; c^{p / m} w_{1} c^{-p / m}, \ldots, c^{p / m} w_{m} c^{-p / m}\right) .
$$

Hence, if $\left(w_{0} ; w_{1}, \ldots, w_{m}\right)$ is fixed by $\phi^{p}$, then each individual $w_{i}$ must be fixed under conjugation by $c^{p / m}$.

Using the notation $W^{\prime}=\operatorname{Cent}_{W}\left(c^{p / m}\right)$, the previous observation means that $w_{i} \in W^{\prime}$, $i=1,2, \ldots, m$. Springer [33, Theorem 4.2] (see also [24, Theorem 11.24(iii)]) proved that $W^{\prime}$ is a well-generated complex reflection group whose degrees coincide with those degrees of $W$ that are divisible by $m h / p$. It was furthermore shown in [9, Lemma 3.3] that

$$
N C(W) \cap W^{\prime}=N C\left(W^{\prime}\right) .
$$


Hence, the tuples $\left(w_{0} ; w_{1}, \ldots, w_{m}\right)$ fixed by $\phi^{p}$ are in fact identical with the elements of $N C^{m}\left(W^{\prime}\right)$, which implies that

$$
\left|\operatorname{Fix}_{N C^{m}(W)}\left(\phi^{p}\right)\right|=\left|N C^{m}\left(W^{\prime}\right)\right| .
$$

Application of Theorem 1 with $W$ replaced by $W^{\prime}$ and of the "limit rule" (5.4) then yields that

$$
\left|N C^{m}\left(W^{\prime}\right)\right|=\prod_{\substack{1 \leq i \leq n \\ \frac{m h}{p} \mid d_{i}}} \frac{m h+d_{i}}{d_{i}}=\left.\operatorname{Cat}^{m}(W ; q)\right|_{q=e^{2 \pi i p / m h}} .
$$

Combining (5.6) and (5.7), we obtain (3.3). This finishes the proof of the lemma.

Lemma 28. Equation (3.3) holds for all divisors $p$ of $m$.

Proof. Using (5.4) and the fact that the degrees of irreducible well-generated complex reflection groups satisfy $d_{i}<h$ for all $i<n$, we see that

$$
\left.\mathrm{Cat}^{m}(W ; q)\right|_{q=e^{2 \pi i p / m h}}= \begin{cases}m+1 & \text { if } m=p, \\ 1 & \text { if } m \neq p .\end{cases}
$$

On the other hand, if $\left(w_{0} ; w_{1}, \ldots, w_{m}\right)$ is fixed by $\phi^{p}$, then, because of the action (5.1), we must have $w_{1}=w_{p+1}=\cdots=w_{m-p+1}$ and $w_{1}=c w_{m-p+1} c^{-1}$. In particular, $w_{1} \in \operatorname{Cent}_{W}(c)$. By the theorem of Springer cited in the proof of Lemma 27, the subgroup $\operatorname{Cent}_{W}(c)$ is itself a complex reflection group whose degrees are those degrees of $W$ that are divisible by $h$. The only such degree is $h$ itself, hence $\operatorname{Cent}_{W}(c)$ is the cyclic group generated by $c$. Moreover, by (5.5), we obtain that $w_{1}=\varepsilon$, the identity element of $W$, or $w_{1}=c$. Therefore, for $m=p$ the set $\operatorname{Fix}_{N C^{m}(W)}\left(\phi^{p}\right)$ consists of the $m+1$ elements $\left(w_{0} ; w_{1}, \ldots, w_{m}\right)$ obtained by choosing $w_{i}=c$ for a particular $i$ between 0 and $m$, all other $w_{j}$ 's being equal to $\varepsilon$, while, for $m \neq p$, we have

$$
\operatorname{Fix}_{N C^{m}(W)}\left(\phi^{p}\right)=\{(c ; \varepsilon, \ldots, \varepsilon)\},
$$

whence the result.

Lemma 29. Let $W$ be an irreducible well-generated complex reflection group all of whose degrees are divisible by $d$. Then each element of $W$ is fixed under conjugation by $c^{h / d}$.

Proof. By the theorem of Springer cited in the proof of Lemma 27, the subgroup $W^{\prime}=$ Cent $_{W}\left(c^{h / d}\right)$ is itself a complex reflection group whose degrees are those degrees of $W$ that are divisible by $d$. Thus, by our assumption, the degrees of $W^{\prime}$ coincide with the degrees of $W$, and hence $W^{\prime}$ must be equal to $W$. Phrased differently, each element of $W$ is fixed under conjugation by $c^{h / d}$, as claimed.

Lemma 30. Let $W$ be an irreducible well-generated complex reflection group of rank $n$, and let $p=m_{1} h_{1}$ be a divisor of $m h$, where $m=m_{1} m_{2}$ and $h=h_{1} h_{2}$. Without loss of generality, we assume that $\operatorname{gcd}\left(h_{1}, m_{2}\right)=1$. Suppose that Theorem 2 has already been verified for all irreducible well-generated complex reflection groups with rank $<n$. If $h_{2}$ does not divide all degrees $d_{i}$, then Equation (3.3) is satisfied. 
Proof. Let us write $h_{1}=a m_{2}+b$, with $0 \leq b<m_{2}$. The condition $\operatorname{gcd}\left(h_{1}, m_{2}\right)=1$ translates into $\operatorname{gcd}\left(b, m_{2}\right)=1$. From (5.1), we infer that

$$
\begin{aligned}
\phi^{p}\left(\left(w_{0} ; w_{1}, \ldots, w_{m}\right)\right) \\
=\left(* ; c^{a+1} w_{m-m_{1} b+1} c^{-a-1}, c^{a+1} w_{m-m_{1} b+2} c^{-a-1}, \ldots, c^{a+1} w_{m} c^{-a-1},\right. \\
\left.c^{a} w_{1} c^{-a}, \ldots, c^{a} w_{m-m_{1} b} c^{-a}\right) .
\end{aligned}
$$

Supposing that $\left(w_{0} ; w_{1}, \ldots, w_{m}\right)$ is fixed by $\phi^{p}$, we obtain the system of equations

$$
\begin{aligned}
& w_{i}=c^{a+1} w_{i+m-m_{1} b} c^{-a-1}, \quad i=1,2, \ldots, m_{1} b, \\
& w_{i}=c^{a} w_{i-m_{1} b} c^{-a}, \quad i=m_{1} b+1, m_{1} b+2, \ldots, m,
\end{aligned}
$$

which, after iteration, implies in particular that

$$
w_{i}=c^{b(a+1)+\left(m_{2}-b\right) a} w_{i} c^{-b(a+1)-\left(m_{2}-b\right) a}=c^{h_{1}} w_{i} c^{-h_{1}}, \quad i=1,2, \ldots, m .
$$

It is at this point where we need $\operatorname{gcd}\left(b, m_{2}\right)=1$. The last equation shows that each $w_{i}$, $i=1,2, \ldots, m$, and thus also $w_{0}$, lies in $\operatorname{Cent}_{W}\left(c^{h_{1}}\right)$. By the theorem of Springer cited in the proof of Lemma 27, this centraliser subgroup is itself a complex reflection group, $W^{\prime}$ say, whose degrees are those degrees of $W$ that are divisible by $h / h_{1}=h_{2}$. Since, by assumption, $h_{2}$ does not divide all degrees, $W^{\prime}$ has rank strictly less than $n$. Again by assumption, we know that Theorem 2 is true for $W^{\prime}$, so that in particular,

$$
\left|\operatorname{Fix}_{N C^{m}\left(W^{\prime}\right)}\left(\phi^{p}\right)\right|=\left.\operatorname{Cat}^{m}\left(W^{\prime} ; q\right)\right|_{q=e^{2 \pi i p / m h}} \text {. }
$$

The arguments above together with (5.5) show that $\operatorname{Fix}_{N C^{m}(W)}\left(\phi^{p}\right)=\operatorname{Fix}_{N C^{m}\left(W^{\prime}\right)}\left(\phi^{p}\right)$. On the other hand, using (5.4) it is straightforward to see that

$$
\left.\mathrm{Cat}^{m}(W ; q)\right|_{q=e^{2 \pi i p / m h}}=\left.\mathrm{Cat}^{m}\left(W^{\prime} ; q\right)\right|_{q=e^{2 \pi i p / m h}} .
$$

This proves (3.3) for our particular $p$, as required.

Lemma 31. Let $W$ be an irreducible well-generated complex reflection group of rank $n$, and let $p=m_{1} h_{1}$ be a divisor of $m h$, where $m=m_{1} m_{2}$ and $h=h_{1} h_{2}$. We assume that $\operatorname{gcd}\left(h_{1}, m_{2}\right)=1$. If $m_{2}>n$ then

$$
\operatorname{Fix}_{N C^{m}(W)}\left(\phi^{p}\right)=\{(c ; \varepsilon, \ldots, \varepsilon)\} .
$$

Proof. Let us suppose that $\left(w_{0} ; w_{1}, \ldots, w_{m}\right) \in \operatorname{Fix}_{N C^{m}(W)}\left(\phi^{p}\right)$ and that there exists a $j \geq 1$ such that $w_{j} \neq \varepsilon$. By (5.8), it then follows for such a $j$ that also $w_{k} \neq \varepsilon$ for all $k \equiv j-l m_{1} b(\bmod m)$, where, as before, $b$ is defined as the unique integer with $h_{1}=a m_{2}+b$ and $0 \leq b<m_{2}$. Since, by assumption, $\operatorname{gcd}\left(b, m_{2}\right)=1$, there are exactly $m_{2}$ such $k$ 's which are distinct $\bmod m$. However, this implies that the sum of the absolute lengths of the $w_{i}$ 's, $0 \leq i \leq m$, is at least $m_{2}>n$, a contradiction to Remark 11.(2).

Remark 3. (1) If we put ourselves in the situation of the assumptions of Lemma 30 , then we may conclude that equation (3.3) only needs to be checked for pairs $\left(m_{2}, h_{2}\right)$ subject to the following restrictions:

$$
m_{2} \geq 2, \quad \operatorname{gcd}\left(h_{1}, m_{2}\right)=1, \quad \text { and } h_{2} \text { divides all degrees of } W .
$$

Indeed, Lemmas 27 and 30 together imply that equation (3.3) is always satisfied in all other cases. 
(2) Still putting ourselves in the situation of Lemma 30, if $m_{2}>n$ and $m_{2} h_{2}$ does not divide any of the degrees of $W$, then equation (3.3) is satisfied. Indeed, Lemma 31 says that in this case the left-hand side of (3.3) equals 1, while a straightforward computation using (5.4) shows that in this case the right-hand side of (3.3) equals 1 as well.

(3) It should be observed that this leaves a finite number of choices for $m_{2}$ to consider, whence a finite number of choices for $\left(m_{1}, m_{2}, h_{1}, h_{2}\right)$. Altogether, there remains a finite number of choices for $p=h_{1} m_{1}$ to be checked.

Lemma 32. Let $W$ be an irreducible well-generated complex reflection group of rank $n$ with the property that $d_{i} \mid h$ for $i=1,2, \ldots, n$. Then Theorem 2 is true for this group $W$.

Proof. By Lemma 26, we may restrict ourselves to divisors $p$ of $m h$.

Suppose that $e^{2 \pi i p / m h}$ is a $d_{i}$-th root of unity for some $i$. In other words, $m h / p$ divides $d_{i}$. Since $d_{i}$ is a divisor of $h$ by assumption, the integer $m h / p$ also divides $h$. But this is equivalent to saying that $m$ divides $p$, and equation (3.3) holds by Lemma 27

Now assume that $m h / p$ does not divide any of the $d_{i}$ 's. Then, by (5.4), the righthand side of (3.3) equals 1 . On the other hand, $(c ; \varepsilon, \ldots, \varepsilon)$ is always an element of $\operatorname{Fix}_{N C^{m}(W)}\left(\phi^{p}\right)$. To see that there are no others, we make appeal to the classification of all irreducible well-generated complex reflection groups, which we recalled in Section 2. Inspection reveals that all groups satisfying the hypotheses of the lemma have rank $n \leq 2$. Except for the groups contained in the infinite series $G(d, 1, n)$ and $G(e, e, n)$ for which Theorem 2 has been established in [19], these are the groups $G_{5}, G_{6}, G_{9}, G_{10}, G_{14}, G_{17}, G_{18}, G_{21}$. We now discuss these groups case by case, keeping the notation of Lemma 30. In order to simplify the argument, we note that Lemma 31 implies that equation (3.3) holds if $m_{2}>2$, so that in the following arguments we always may assume that $m_{2}=2$.

CASE $G_{5}$. The degrees are 6,12, and therefore Remark 3.(1) implies that equation (3.3) is always satisfied.

CASE $G_{6}$. The degrees are 4,12, and therefore, according to Remark 3. (1), we need only consider the case where $h_{2}=4$ and $m_{2}=2$, that is, $p=3 \mathrm{~m} / 2$. Then (5.8) becomes

$$
\phi^{p}\left(\left(w_{0} ; w_{1}, \ldots, w_{m}\right)\right)=\left(* ; c^{2} w_{\frac{m}{2}+1} c^{-2}, c^{2} w_{\frac{m}{2}+2} c^{-2}, \ldots, c^{2} w_{m} c^{-2}, c w_{1} c^{-1}, \ldots, c w_{\frac{m}{2}} c^{-1}\right) .
$$

If $\left(w_{0} ; w_{1}, \ldots, w_{m}\right)$ is fixed by $\phi^{p}$ and not equal to $(c ; \varepsilon, \ldots, \varepsilon)$, there must exist an $i$ with $1 \leq i \leq \frac{m}{2}$ such that $\ell_{T}\left(w_{i}\right)=\ell_{T}\left(w_{\frac{m}{2}+i}\right)=1, w_{\frac{m}{2}+i}=c w_{i} c^{-1}, w_{i} w_{\frac{m}{2}+i}=w_{i} c w_{i} c^{-1}=c$, and all $w_{j}$, with $j \neq i, \frac{m}{2}+i$, equal $\varepsilon$. However, with the help of the GAP package CHEVIE [14, 28], one verifies that there is no $w_{i}$ in $G_{6}$ such that

$$
\ell_{T}\left(w_{i}\right)=1 \text { and } w_{i} c w_{i} c^{-1}=c
$$

are simultaneously satisfied. Hence, the left-hand side of (3.3) is equal to 1, as required.

CASE $G_{9}$. The degrees are 8, 24, and therefore, according to Remark 3. (1), we need only consider the case where $h_{2}=8$ and $m_{2}=2$, that is, $p=3 m / 2$. This is the same $p$ as for $G_{6}$. Again, CHEVIE finds no solution. Hence, the left-hand side of (3.3) is equal to 1 , as required.

CASE $G_{10}$. The degrees are 12,24, and therefore Remark 3.(1) implies that equation (3.3) is always satisfied. 
CAse $G_{14}$. The degrees are 6,24, and therefore Remark 3,(1) implies that equation (3.3) is always satisfied.

CAsE $G_{17}$. The degrees are 20,60, and therefore, according to Remark 3 . (1), we need only consider the cases where $h_{2}=20$ or $h_{2}=4$. In the first case, $p=3 \mathrm{~m} / 2$, which is the same $p$ as for $G_{6}$. Again, CHEVIE finds no solution. In the second case, $p=15 \mathrm{~m} / 2$. Then (5.8) becomes

$$
\begin{aligned}
\phi^{p}\left(\left(w_{0} ; w_{1}, \ldots, w_{m}\right)\right) \\
\quad=\left(* ; c^{8} w_{\frac{m}{2}+1} c^{-8}, c^{8} w_{\frac{m}{2}+2} c^{-8}, \ldots, c^{8} w_{m} c^{-8}, c^{7} w_{1} c^{-7}, \ldots, c^{7} w_{\frac{m}{2}} c^{-7}\right) .
\end{aligned}
$$

By Lemma 29, every element of $N C(W)$ is fixed under conjugation by $c^{3}$, and, thus, on elements fixed by $\phi^{p}$, the above action of $\phi^{p}$ reduces to the one in (5.10). This action was already discussed in the first case. Hence, in both cases, the left-hand side of (3.3) is equal to 1 , as required.

CAsE $G_{18}$. The degrees are 30,60, and therefore Remark 3.(1) implies that equation (3.3) is always satisfied.

CAsE $G_{21}$. The degrees are 12,60, and therefore, according to Remark 3. (1), we need only consider the cases where $h_{2}=12$ or $h_{2}=4$. In the first case, $p=5 \mathrm{~m} / 2$, so that (5.8) becomes

$$
\begin{aligned}
\phi^{p}\left(\left(w_{0} ; w_{1}, \ldots, w_{m}\right)\right) \\
\quad=\left(* ; c^{3} w_{\frac{m}{2}+1} c^{-3}, c^{3} w_{\frac{m}{2}+2} c^{-3}, \ldots, c^{3} w_{m} c^{-3}, c^{2} w_{1} c^{-2}, \ldots, c^{2} w_{\frac{m}{2}} c^{-2}\right) .
\end{aligned}
$$

If $\left(w_{0} ; w_{1}, \ldots, w_{m}\right)$ is fixed by $\phi^{p}$ and not equal to $(c ; \varepsilon, \ldots, \varepsilon)$, there must exist an $i$ with $1 \leq i \leq \frac{m}{2}$ such that $\ell_{T}\left(w_{i}\right)=1$ and $w_{i} c^{2} w_{i} c^{-2}=c$. However, with the help of the GAP package CHEVIE [14, 28], one verifies that there is no such solution to this equation. In the second case, $p=15 \mathrm{~m} / 2$. Then (5.8) becomes the action in (5.11). By Lemma 29, every element of $N C(W)$ is fixed under conjugation by $c^{5}$, and, thus, on elements fixed by $\phi^{p}$, the action of $\phi^{p}$ in (5.11) reduces to the one in the first case. Hence, in both cases, the left-hand side of (3.3) is equal to 1, as required.

This completes the proof of the lemma.

\section{Exemplification of CASE-By-CASE Verification of Theorem 2}

It remains to verify Theorem 2 for the groups $G_{4}, G_{8}, G_{16}, G_{20}, G_{23}=H_{3}, G_{24}, G_{25}$, $G_{26}, G_{27}, G_{28}=F_{4}, G_{29}, G_{30}=H_{4}, G_{32}, G_{33}, G_{34}, G_{35}=E_{6}, G_{36}=E_{7}, G_{37}=E_{8}$. All details can be found in [21, Sec. 6]. We content ourselves with illustrating the type of computation that is needed here by going through the case of the group $G_{24}$, and by discussing some of the arguments needed for the group $G_{37}=E_{8}$.

In the sequel we write $\zeta_{d}$ for a primitive $d$-th root of unity.

CAsE $G_{24}$. The degrees are 4,6,14, and hence we have

$$
\operatorname{Cat}^{m}\left(G_{24} ; q\right)=\frac{[14 m+14]_{q}[14 m+6]_{q}[14 m+4]_{q}}{[14]_{q}[6]_{q}[4]_{q}}
$$

Let $\zeta$ be a $14 m$-th root of unity. In what follows, we abbreviate the assertion that " $\zeta$ is a primitive $d$-th root of unity" as " $\zeta=\zeta_{d}$." The following cases on the right-hand side 
of (3.3) occur:

$$
\begin{aligned}
& \lim _{q \rightarrow \zeta} \operatorname{Cat}^{m}\left(G_{24} ; q\right)=m+1, \quad \text { if } \zeta=\zeta_{14}, \zeta_{7}, \\
& \lim _{q \rightarrow \zeta} \operatorname{Cat}^{m}\left(G_{24} ; q\right)=\frac{7 m+3}{3}, \quad \text { if } \zeta=\zeta_{6}, \zeta_{3}, 3 \mid m, \\
& \lim _{q \rightarrow \zeta} \operatorname{Cat}^{m}\left(G_{24} ; q\right)=\frac{7 m+2}{2}, \quad \text { if } \zeta=\zeta_{4}, 2 \mid m, \\
& \lim _{q \rightarrow \zeta} \operatorname{Cat}^{m}\left(G_{24} ; q\right)=\operatorname{Cat}^{m}\left(G_{24}\right), \quad \text { if } \zeta=-1 \text { or } \zeta=1, \\
& \lim _{q \rightarrow \zeta} \operatorname{Cat}^{m}\left(G_{24} ; q\right)=1, \quad \text { otherwise. }
\end{aligned}
$$

We must now prove that the left-hand side of (3.3) in each case agrees with the values exhibited in (6.1). The only cases not covered by Lemma 27 are the ones in (6.1b), (6.1c), and (6.1e). (In both (6.1a) and (6.1d ) we have $d \mid h$.

We first consider (6.1b). By Lemma 26, we are free to choose $p=7 m / 3$ if $\zeta=\zeta_{6}$, respectively $p=14 m / 3$ if $\zeta=\zeta_{3}$. In both cases, $m$ must be divisible by 3 .

We start with the case that $p=7 \mathrm{~m} / 3$. From (5.1), we infer

$$
\begin{aligned}
\phi^{p}\left(\left(w_{0} ; w_{1}, \ldots,\right.\right. & \left.\left.w_{m}\right)\right) \\
& =\left(* ; c^{3} w_{\frac{2 m}{3}+1} c^{-3}, c^{3} w_{\frac{2 m}{3}+2} c^{-3}, \ldots, c^{3} w_{m} c^{-3}, c^{2} w_{1} c^{-2}, \ldots, c^{2} w_{\frac{2 m}{3}} c^{-2}\right) .
\end{aligned}
$$

Supposing that $\left(w_{0} ; w_{1}, \ldots, w_{m}\right)$ is fixed by $\phi^{p}$, we obtain the system of equations

$$
\begin{aligned}
& w_{i}=c^{3} w_{\frac{2 m}{3}+i} c^{-3}, \quad i=1,2, \ldots, \frac{m}{3}, \\
& w_{i}=c^{2} w_{i-\frac{m}{3}} c^{-2}, \quad i=\frac{m}{3}+1, \frac{m}{3}+2, \ldots, m .
\end{aligned}
$$

There are two distinct possibilities for choosing the $w_{i}$ 's, $1 \leq i \leq m$ : either all the $w_{i}$ 's are equal to $\varepsilon$, or there is an $i$ with $1 \leq i \leq \frac{m}{3}$ such that

$$
\ell_{T}\left(w_{i}\right)=\ell_{T}\left(w_{i+\frac{m}{3}}\right)=\ell_{T}\left(w_{i+\frac{2 m}{3}}\right)=1 .
$$

Writing $t_{1}, t_{2}, t_{3}$ for $w_{i}, w_{i+\frac{m}{3}}, w_{i+\frac{2 m}{3}}$, respectively, the equations (6.2) reduce to

$$
\begin{aligned}
& t_{1}=c^{3} t_{3} c^{-3}, \\
& t_{2}=c^{2} t_{1} c^{-2}, \\
& t_{3}=c^{2} t_{2} c^{-2} .
\end{aligned}
$$

One of these equations is in fact superfluous: if we substitute (6.3b) and (6.3c) in (6.3a), then we obtain $t_{1}=c^{7} t_{1} c^{-7}$ which is automatically satisfied due to Lemma 29 with $d=2$.

Since $\left(w_{0} ; w_{1}, \ldots, w_{m}\right) \in N C^{m}\left(G_{24}\right)$, we must have $t_{1} t_{2} t_{3}=c$. Combining this with (6.3), we infer that

$$
t_{1}\left(c^{2} t_{1} c^{-2}\right)\left(c^{4} t_{1} c^{-4}\right)=c .
$$

With the help of CHEVIE, one obtains 7 solutions for $t_{1}$ in this equation, each of them giving rise to $m / 3$ elements of $\operatorname{Fix}_{N C^{m}\left(G_{24}\right)}\left(\phi^{p}\right)$ since $i$ (in $\left.w_{i}\right)$ ranges from 1 to $m / 3$.

In total, we obtain $1+7 \frac{m}{3}=\frac{7 m+3}{3}$ elements in $\operatorname{Fix}_{N C^{m}\left(G_{24}\right)}\left(\phi^{p}\right)$, which agrees with the limit in $6.1 \mathrm{~b}$.

The case where $p=14 m / 3$ can be treated in a similar fashion. In the end, it turns out that we have to solve the same enumeration problem as for $p=7 \mathrm{~m} / 3$, and, 
consequently, the number of elements of $\operatorname{Fix}_{N C^{m}\left(G_{24}\right)}\left(\phi^{p}\right)$ is the same, namely $\frac{7 m+3}{3}$, as required.

Our next case is (6.1c). Proceeding in a similar manner as before, we see that there is again the trivial possibility $(c ; \varepsilon, \ldots, \varepsilon)$, and otherwise we have to find $t_{1}$ with $\ell_{T}\left(t_{1}\right)=1$ satisfying the inequality

$$
t_{1}\left(c^{3} t_{1} c^{-3}\right) \leq_{T} c .
$$

With the help of CHEVIE, one obtains 7 solutions for $t_{1}$ in this relation, each of them giving rise to $m / 2$ elements of $\operatorname{Fix}_{N C^{m}\left(G_{24}\right)}\left(\phi^{p}\right)$ since $i$ (in $w_{i}$ ) ranges from 1 to $m / 2$.

In total, we obtain $1+7 \frac{m}{2}=\frac{7 m+2}{2}$ elements in $\operatorname{Fix}_{N C^{m}\left(G_{24}\right)}\left(\phi^{p}\right)$, which agrees with the limit in (6.1c).

Finally, we turn to (6.1e). By Remark 3, the only choices for $h_{2}$ and $m_{2}$ to be considered are $h_{2}=1$ and $m_{2}=3, h_{2}=m_{2}=2$, and $h_{2}=2$ and $m_{2}=3$. These correspond to the choices $p=14 \mathrm{~m} / 3, p=7 \mathrm{~m} / 2$, respectively $p=7 \mathrm{~m} / 3$, all of which have already been discussed as they do not belong to (6.1e). Hence, (3.3) must necessarily hold, as required.

CASE $G_{37}=E_{8}$. The degrees are 2, 8, 12, 14, 18, 20, 24, 30, and hence we have

$$
\begin{aligned}
\operatorname{Cat}^{m}\left(E_{8} ; q\right)=\frac{[30 m+30]_{q}[30 m+24]_{q}[30 m+20]_{q}[30 m+18]_{q}}{[30]_{q}[24]_{q}[20]_{q}[18]_{q}} \\
\times \frac{[30 m+14]_{q}[30 m+12]_{q}[30 m+8]_{q}[30 m+2]_{q}}{[14]_{q}[12]_{q}[8]_{q}[2]_{q}} .
\end{aligned}
$$

Let $\zeta$ be a $30 \mathrm{~m}$-th root of unity. The cases occurring on the right-hand side of (3.3) not covered by Lemma 27 are:

$$
\begin{aligned}
& \lim _{q \rightarrow \zeta} \operatorname{Cat}^{m}\left(E_{8} ; q\right)=\frac{5 m+4}{4}, \quad \text { if } \zeta=\zeta_{24}, 4 \mid m, \\
& \lim _{q \rightarrow \zeta} \operatorname{Cat}^{m}\left(E_{8} ; q\right)=\frac{3 m+2}{2}, \quad \text { if } \zeta=\zeta_{20}, 2 \mid m, \\
& \lim _{q \rightarrow \zeta} \operatorname{Cat}^{m}\left(E_{8} ; q\right)=\frac{5 m+3}{3}, \quad \text { if } \zeta=\zeta_{18}, \zeta_{9}, 3 \mid m, \\
& \lim _{q \rightarrow \zeta} \operatorname{Cat}^{m}\left(E_{8} ; q\right)=\frac{15 m+7}{7}, \quad \text { if } \zeta=\zeta_{14}, \zeta_{7}, 7 \mid m, \\
& \lim _{q \rightarrow \zeta} \operatorname{Cat}^{m}\left(E_{8} ; q\right)=\frac{(5 m+4)(5 m+2)}{8}, \quad \text { if } \zeta=\zeta_{12}, 2 \mid m, \\
& \lim _{q \rightarrow \zeta} \operatorname{Cat}^{m}\left(E_{8} ; q\right)=\frac{(5 m+4)(15 m+4)}{16}, \quad \text { if } \zeta=\zeta_{8}, 4 \mid m, \\
& \lim _{q \rightarrow \zeta} \operatorname{Cat}^{m}\left(E_{8} ; q\right)=\frac{(5 m+4)(3 m+2)(5 m+2)(15 m+4)}{64}, \quad \text { if } \zeta=\zeta_{4}, 2 \mid m, \\
& \lim _{q \rightarrow \zeta} \operatorname{Cat}^{m}\left(E_{8} ; q\right)=\operatorname{Cat}^{m}\left(E_{8}\right), \quad \text { if } \zeta=-1 \text { or } \zeta=1, \\
& \lim _{q \rightarrow \zeta} \operatorname{Cat}^{m}\left(E_{8} ; q\right)=1, \quad \text { otherwise. }
\end{aligned}
$$

We now have to prove that the left-hand side of (3.3) in each case agrees with the values exhibited in (6.6). Since the corresponding computations in the various cases are very similar, we concentrate here only on the cases (6.6f) and (6.6g), these two being representative of the types of arguments arising. As before, we refer the reader to [21, Sec. 6] for full details. 
Let us consider the case in (6.6f) first. By Lemma 26, we are free to choose $p=15 \mathrm{~m} / 4$. In particular, $m$ must be divisible by 4 . From (5.1), we infer

$$
\begin{aligned}
& \phi^{p}\left(\left(w_{0} ; w_{1}, \ldots, w_{m}\right)\right) \\
& \quad=\left(* ; c^{4} w_{\frac{m}{4}+1} c^{-4}, c^{4} w_{\frac{m}{4}+2} c^{-4}, \ldots, c^{4} w_{m} c^{-4}, c^{3} w_{1} c^{-3}, \ldots, c^{3} w_{\frac{m}{4}} c^{-3}\right) .
\end{aligned}
$$

Supposing that $\left(w_{0} ; w_{1}, \ldots, w_{m}\right)$ is fixed by $\phi^{p}$, we obtain the system of equations

$$
\begin{aligned}
& w_{i}=c^{4} w_{\frac{m}{4}+i} c^{-4}, \quad i=1,2, \ldots, \frac{3 m}{4}, \\
& w_{i}=c^{3} w_{i-\frac{3 m}{4}} c^{-3}, \quad i=\frac{3 m}{4}+1, \frac{3 m}{4}+2, \ldots, m .
\end{aligned}
$$

There are several distinct possibilities for choosing the $w_{i}$ 's, $1 \leq i \leq m$, which we summarise as follows:

(i) all the $w_{i}$ 's are equal to $\varepsilon$ (and $w_{0}=c$ ),

(ii) there is an $i$ with $1 \leq i \leq \frac{m}{4}$ such that

$$
1 \leq \ell_{T}\left(w_{i}\right)=\ell_{T}\left(w_{i+\frac{m}{4}}\right)=\ell_{T}\left(w_{i+\frac{2 m}{4}}\right)=\ell_{T}\left(w_{i+\frac{3 m}{4}}\right) \leq 2,
$$

and the other $w_{j}$ 's, $1 \leq j \leq m$, are equal to $\varepsilon$,

(iii) there are $i_{1}$ and $i_{2}$ with $1 \leq i_{1}<i_{2} \leq \frac{m}{4}$ such that

$$
\begin{aligned}
\ell_{T}\left(w_{i_{1}}\right)=\ell_{T}\left(w_{i_{2}}\right)=\ell_{T}\left(w_{i_{1}+\frac{m}{4}}\right)=\ell_{T}\left(w_{i_{2}+\frac{m}{4}}\right) & \\
= & \ell_{T}\left(w_{i_{1}+\frac{2 m}{4}}\right)=\ell_{T}\left(w_{i_{2}+\frac{2 m}{4}}\right)=\ell_{T}\left(w_{i_{1}+\frac{3 m}{4}}\right)=\ell_{T}\left(w_{i_{2}+\frac{3 m}{4}}\right)=1,
\end{aligned}
$$

and all other $w_{j}$ are equal to $\varepsilon$.

Moreover, since $\left(w_{0} ; w_{1}, \ldots, w_{m}\right) \in N C^{m}\left(E_{8}\right)$, we must have

$$
w_{i} w_{i+\frac{m}{4}} w_{i+\frac{2 m}{4}} w_{i+\frac{3 m}{4}} \leq_{T} c
$$

or

$$
w_{i_{1}} w_{i_{2}} w_{i_{1}+\frac{m}{4}} w_{i_{2}+\frac{m}{4}} w_{i_{1}+\frac{2 m}{4}} w_{i_{2}+\frac{2 m}{4}} w_{i_{1}+\frac{3 m}{4}} w_{i_{2}+\frac{3 m}{4}}=c .
$$

Together with equations (6.7)-(6.8), this implies that

$$
w_{i}=c^{15} w_{i} c^{-15} \text { and } w_{i}\left(c^{11} w_{i} c^{-11}\right)\left(c^{7} w_{i} c^{-7}\right)\left(c^{3} w_{i} c^{-3}\right) \leq_{T} c
$$

or that

$$
\begin{aligned}
& w_{i_{1}}=c^{15} w_{i_{1}} c^{-15}, \quad w_{i_{1}}=c^{15} w_{i_{2}} c^{-15} \\
& \quad \text { and } w_{i_{1}} w_{i_{2}}\left(c^{11} w_{i_{1}} c^{-11}\right)\left(c^{11} w_{i_{2}} c^{-11}\right)\left(c^{7} w_{i_{1}} c^{-7}\right)\left(c^{7} w_{i_{2}} c^{-7}\right)\left(c^{3} w_{i_{1}} c^{-3}\right)\left(c^{3} w_{i_{2}} c^{-3}\right)=c .
\end{aligned}
$$

Here, the first equation in (6.9) and the first two equations in (6.10) are automatically satisfied due to Lemma 29 with $d=2$.

With the help of Stembridge's Maple package coxeter [36], one obtains 30 solutions for $w_{i}$ in (6.9) with $\ell_{T}\left(w_{i}\right)=1,45$ solutions for $w_{i}$ with $\ell_{T}\left(w_{i}\right)=2$ and $w_{i}$ of type $A_{1}^{2}$ (as a parabolic Coxeter element; see the end of Section 21), and 20 solutions for $w_{i}$ with $\ell_{T}\left(w_{i}\right)=2$ and $w_{i}$ of type $A_{2}$. Each of them gives rise to $m / 4$ elements of $\operatorname{Fix}_{N C^{m}\left(E_{8}\right)}\left(\phi^{p}\right)$ since $i$ ranges from 1 to $m / 4$.

The number of solutions in Case (iii) can be computed from our knowledge of the solutions in Case (ii) according to type, using some elementary counting arguments. Namely, the number of solutions of (6.10) is equal to

$$
45 \cdot 2+20 \cdot 3=150,
$$


since an element of type $A_{1}^{2}$ can be decomposed in two ways into a product of two elements of absolute length 1, while for an element of type $A_{2}$ this can be done in 3 ways.

In total, we obtain $1+(30+45+20) \frac{m}{4}+150\left(\begin{array}{c}m / 4 \\ 2\end{array}\right)=\frac{(5 m+4)(15 m+4)}{16}$ elements in $\operatorname{Fix}_{N C^{m}\left(E_{8}\right)}\left(\phi^{p}\right)$, which agrees with the limit in (6.6f).

Next, we discuss the case in (6.6g). By Lemma 26, we are free to choose $p=15 \mathrm{~m} / 2$. In particular, $m$ must be divisible by 2. From (5.1), we infer

$$
\begin{aligned}
& \phi^{p}\left(\left(w_{0} ; w_{1}, \ldots, w_{m}\right)\right) \\
& \quad=\left(* ; c^{8} w_{\frac{m}{2}+1} c^{-8}, c^{8} w_{\frac{m}{2}+2} c^{-8}, \ldots, c^{8} w_{m} c^{-8}, c^{7} w_{1} c^{-7}, \ldots, c^{7} w_{\frac{m}{2}} c^{-7}\right) .
\end{aligned}
$$

Supposing that $\left(w_{0} ; w_{1}, \ldots, w_{m}\right)$ is fixed by $\phi^{p}$, we obtain the system of equations

$$
\begin{array}{ll}
w_{i}=c^{8} w_{\frac{m}{2}+i} c^{-8}, & i=1,2, \ldots, \frac{m}{2}, \\
w_{i}=c^{7} w_{i-\frac{m}{2}} c^{-7}, & i=\frac{m}{2}+1, \frac{m}{2}+2, \ldots, m .
\end{array}
$$

There are several distinct possibilities for choosing the $w_{i}$ 's, $1 \leq i \leq m$ :

(i) all the $w_{i}$ 's are equal to $\varepsilon$ (and $w_{0}=c$ ),

(ii) there is an $i$ with $1 \leq i \leq \frac{m}{2}$ such that

$$
1 \leq \ell_{T}\left(w_{i}\right)=\ell_{T}\left(w_{i+\frac{m}{2}}\right) \leq 4
$$

and the other $w_{j}$ 's, $1 \leq j \leq m$, are equal to $\varepsilon$,

(iii) there are $i_{1}$ and $i_{2}$ with $1 \leq i_{1}<i_{2} \leq \frac{m}{2}$ such that

$$
\ell_{1}:=\ell_{T}\left(w_{i_{1}}\right)=\ell_{T}\left(w_{i_{1}+\frac{m}{2}}\right) \geq 1, \quad \ell_{2}:=\ell_{T}\left(w_{i_{2}}\right)=\ell_{T}\left(w_{i_{2}+\frac{m}{2}}\right) \geq 1, \quad \text { and } \quad \ell_{1}+\ell_{2} \leq 4 \text {, }
$$

and the other $w_{j}$ 's, $1 \leq j \leq m$, are equal to $\varepsilon$,

(iv) there are $i_{1}, i_{2}, i_{3}$ with $1 \leq i_{1}<i_{2}<i_{3} \leq \frac{m}{2}$ such that

$$
\begin{aligned}
& \ell_{1}:=\ell_{T}\left(w_{i_{1}}\right)=\ell_{T}\left(w_{i_{1}+\frac{m}{2}}\right) \geq 1, \quad \ell_{2}:=\ell_{T}\left(w_{i_{2}}\right)=\ell_{T}\left(w_{i_{2}+\frac{m}{2}}\right) \geq 1, \\
& \ell_{3}:=\ell_{T}\left(w_{i_{3}}\right)=\ell_{T}\left(w_{i_{3}+\frac{m}{2}}\right) \geq 1, \quad \text { and } \quad \ell_{1}+\ell_{2}+\ell_{3} \leq 4,
\end{aligned}
$$

and the other $w_{j}$ 's, $1 \leq j \leq m$, are equal to $\varepsilon$,

(v) there are $i_{1}, i_{2}, i_{3}, i_{4}$ with $1 \leq i_{1}<i_{2}<i_{3}<i_{4} \leq \frac{m}{2}$ such that

$$
\begin{aligned}
\ell_{T}\left(w_{i_{1}}\right)=\ell_{T}\left(w_{i_{2}}\right) & =\ell_{T}\left(w_{i_{3}}\right)=\ell_{T}\left(w_{i_{4}}\right) \\
& =\ell_{T}\left(w_{i_{1}+\frac{m}{2}}\right)=\ell_{T}\left(w_{i_{2}+\frac{m}{2}}\right)=\ell_{T}\left(w_{i_{3}+\frac{m}{2}}\right)=\ell_{T}\left(w_{i_{4}+\frac{m}{2}}\right)=1,
\end{aligned}
$$

and all other $w_{j}$ 's are equal to $\varepsilon$.

Moreover, since $\left(w_{0} ; w_{1}, \ldots, w_{m}\right) \in N C^{m}\left(E_{8}\right)$, we must have $w_{i} w_{i+\frac{m}{2}} \leq_{T} c$, respectively $w_{i_{1}} w_{i_{2}} w_{i_{1}+\frac{m}{2}} w_{i_{2}+\frac{m}{2}} \leq_{T} c$, respectively

$$
w_{i_{1}} w_{i_{2}} w_{i_{3}} w_{i_{1}+\frac{m}{2}} w_{i_{2}+\frac{m}{2}} w_{i_{3}+\frac{m}{2}} \leq_{T} c
$$

respectively

$$
w_{i_{1}} w_{i_{2}} w_{i_{3}} w_{i_{4}} w_{i_{1}+\frac{m}{2}} w_{i_{2}+\frac{m}{2}} w_{i_{3}+\frac{m}{2}} w_{i_{4}+\frac{m}{2}}=c .
$$

Together with equations (6.11)-6.12), this implies that

$$
w_{i}=c^{15} w_{i} c^{-15} \text { and } w_{i}\left(c^{7} w_{i} c^{-7}\right) \leq_{T} c,
$$

respectively that

$$
w_{i_{1}}=c^{15} w_{i_{1}} c^{-15}, \quad w_{i_{2}}=c^{15} w_{i_{2}} c^{-15}, \quad \text { and } \quad w_{i_{1}} w_{i_{2}}\left(c^{7} w_{i_{1}} c^{-7}\right)\left(c^{7} w_{i_{2}} c^{-7}\right) \leq_{T} c,
$$


respectively that

$$
\begin{aligned}
w_{i_{1}}=c^{15} w_{i_{1}} c^{-15}, \quad w_{i_{2}}= & c^{15} w_{i_{2}} c^{-15}, \quad w_{i_{3}}=c^{15} w_{i_{3}} c^{-15}, \\
& \text { and } w_{i_{1}} w_{i_{2}} w_{i_{3}}\left(c^{7} w_{i_{1}} c^{-7}\right)\left(c^{7} w_{i_{2}} c^{-7}\right)\left(c^{7} w_{i_{3}} c^{-7}\right) \leq_{T} c,
\end{aligned}
$$

respectively that

$$
\begin{aligned}
w_{i_{1}}=c^{15} w_{i_{1}} c^{-15}, & w_{i_{2}}=c^{15} w_{i_{2}} c^{-15}, \quad w_{i_{3}}=c^{15} w_{i_{3}} c^{-15}, \quad w_{i_{4}}=c^{15} w_{i_{4}} c^{-15}, \\
\text { and } \quad & w_{i_{1}} w_{i_{2}} w_{i_{3}} w_{i_{4}}\left(c^{7} w_{i_{1}} c^{-7}\right)\left(c^{7} w_{i_{2}} c^{-7}\right)\left(c^{7} w_{i_{3}} c^{-7}\right)\left(c^{7} w_{i_{4}} c^{-7}\right)=c .
\end{aligned}
$$

Here, the first equation in (6.13), the first two in (6.14), the first three in (6.15), and the first four in (6.16), are all automatically satisfied due to Lemma 29 with $d=2$.

With the help of Stembridge's Maple package coxeter [36], one obtains

- 45 solutions for $w_{i}$ in (6.13) with $\ell_{T}\left(w_{i}\right)=1$,

- 150 solutions for $w_{i}$ in (6.13) with $\ell_{T}\left(w_{i}\right)=2$ and $w_{i}$ of type $A_{1}^{2}$,

- 100 solutions for $w_{i}$ in (6.13) with $\ell_{T}\left(w_{i}\right)=2$ and $w_{i}$ of type $A_{2}$,

- 75 solutions for $w_{i}$ in (6.13) with $\ell_{T}\left(w_{i}\right)=3$ and $w_{i}$ of type $A_{1}^{3}$,

- 165 solutions for $w_{i}$ in (6.13) with $\ell_{T}\left(w_{i}\right)=3$ and $w_{i}$ of type $A_{1} * A_{2}$,

- 90 solutions for $w_{i}$ in (6.13) with $\ell_{T}\left(w_{i}\right)=3$ and $w_{i}$ of type $A_{3}$,

- 15 solutions for $w_{i}$ in (6.13) with $\ell_{T}\left(w_{i}\right)=4$ and $w_{i}$ of type $A_{1}^{2} * A_{2}$,

- 45 solutions for $w_{i}$ in (6.13) with $\ell_{T}\left(w_{i}\right)=4$ and $w_{i}$ of type $A_{1} * A_{3}$;

- 5 solutions for $w_{i}$ in (6.13) with $\ell_{T}\left(w_{i}\right)=4$ and $w_{i}$ of type $A_{2}^{2}$,

- 18 solutions for $w_{i}$ in (6.13) with $\ell_{T}\left(w_{i}\right)=4$ and $w_{i}$ of type $A_{4}$,

- 5 solutions for $w_{i}$ in (6.13) with $\ell_{T}\left(w_{i}\right)=4$ and $w_{i}$ of type $D_{4}$.

Each of them gives rise to $m / 2$ elements of $\operatorname{Fix}_{N C^{m}\left(E_{8}\right)}\left(\phi^{p}\right)$ since $i$ ranges from 1 to $m / 2$. There are no solutions for $w_{i}$ in (6.13) with $w_{i}$ of type $A_{1}^{4}$.

Letting the computer find all solutions in cases (iii)-(v) would take years. However, the number of these solutions can be computed from our knowledge of the solutions in Case (ii) according to type, if this information is combined with the decomposition numbers in the sense of [17, 18, 20] (see the end of Section 2) and some elementary (multiset) permutation counting. The decomposition numbers for $A_{2}, A_{3}, A_{4}$, and $D_{4}$ of which we make use can be found in the appendix of [18].

To begin with, the number of solutions of (6.14) with $\ell_{1}=\ell_{2}=1$ is equal to

$$
n_{1,1}:=150 \cdot 2+100 \cdot N_{A_{2}}\left(A_{1}, A_{1}\right)=600,
$$

since an element of type $A_{1}^{2}$ can be decomposed in two ways into a product of two elements of absolute length 1 , while for an element of type $A_{2}$ this can be done in $N_{A_{2}}\left(A_{1}, A_{1}\right)=3$ ways. Similarly, the number of solutions of (6.14) with $\ell_{1}=2$ and $\ell_{2}=1$ is equal to

$$
n_{2,1}:=75 \cdot 3+165 \cdot\left(1+N_{A_{2}}\left(A_{1}, A_{1}\right)\right)+90 \cdot N_{A_{3}}\left(A_{2}, A_{1}\right)=1425,
$$

the number of solutions of (6.14) with $\ell_{1}=3$ and $\ell_{2}=1$ is equal to

$$
\begin{aligned}
& n_{3,1}:=15 \cdot\left(2+N_{A_{2}}\left(A_{1}, A_{1}\right)\right)+45 \cdot\left(1+N_{A_{3}}\left(A_{2}, A_{1}\right)\right)+5 \cdot\left(2 N_{A_{2}}\left(A_{1}, A_{1}\right)\right) \\
& \quad+18 \cdot\left(N_{A_{4}}\left(A_{3}, A_{1}\right)+N_{A_{4}}\left(A_{1} * A_{2}, A_{1}\right)\right)+5 \cdot\left(N_{D_{4}}\left(A_{3}, A_{1}\right)+N_{D_{4}}\left(A_{1}^{3}, A_{1}\right)\right)=660,
\end{aligned}
$$


the number of solutions of (6.14) with $\ell_{1}=\ell_{2}=2$ is equal to

$$
\begin{aligned}
n_{2,2}:=15 \cdot(2+ & \left.2 N_{A_{2}}\left(A_{1}, A_{1}\right)\right)+45 \cdot\left(2 N_{A_{3}}\left(A_{2}, A_{1}\right)\right)+5 \cdot\left(2+N_{A_{2}}\left(A_{1}, A_{1}\right)^{2}\right) \\
+ & 18 \cdot\left(N_{A_{4}}\left(A_{2}, A_{2}\right)+\right. \\
& \left.N_{A_{4}}\left(A_{1}^{2}, A_{1}^{2}\right)+2 N_{A_{4}}\left(A_{2}, A_{1}^{2}\right)\right) \\
& +5 \cdot\left(N_{D_{4}}\left(A_{2}, A_{2}\right)+2 N_{D_{4}}\left(A_{2}, A_{1}^{2}\right)\right)=1195,
\end{aligned}
$$

the number of solutions of (6.15) with $\ell_{1}=\ell_{2}=\ell_{3}=1$ is equal to

$$
n_{1,1,1}:=75 \cdot 3 !+165 \cdot\left(3 N_{A_{2}}\left(A_{1}, A_{1}\right)\right)+90 N_{A_{3}}\left(A_{1}, A_{1}, A_{1}\right)=3375,
$$

the number of solutions of (6.15) with $\ell_{1}=2$ and $\ell_{2}=\ell_{3}=1$ is equal to

$$
\begin{array}{r}
n_{2,1,1}:=15 \cdot\left(2+N_{A_{2}}\left(A_{1}, A_{1}\right)+2 \cdot 2 \cdot N_{A_{2}}\left(A_{1}, A_{1}\right)\right)+45 \cdot\left(2 N_{A_{3}}\left(A_{2}, A_{1}\right)+N_{A_{3}}\left(A_{1}, A_{1}, A_{1}\right)\right) \\
+5 \cdot\left(2 N_{A_{2}}\left(A_{1}, A_{1}\right)+2 N_{A_{2}}\left(A_{1}, A_{1}\right)^{2}\right)+18 \cdot\left(N_{A_{4}}\left(A_{2}, A_{1}, A_{1}\right)+N_{A_{4}}\left(A_{1}^{2}, A_{1}, A_{1}\right)\right) \\
+5 \cdot\left(N_{D_{4}}\left(A_{2}, A_{1}, A_{1}\right)+N_{D_{4}}\left(A_{1}^{2}, A_{1}, A_{1}\right)\right)=2850
\end{array}
$$

and the number of solutions of (6.16) is equal to

$$
\begin{aligned}
n_{1,1,1,1}:=15 \cdot\left(12 N_{A_{2}}\left(A_{1}, A_{1}\right)\right)+45 \cdot\left(4 N_{A_{3}}\left(A_{1}, A_{1}, A_{1}\right)\right)+5 \cdot\left(6 N_{A_{2}}\left(A_{1}, A_{1}\right)^{2}\right) \\
+18 \cdot N_{A_{4}}\left(A_{1}, A_{1}, A_{1}, A_{1}\right)+5 \cdot N_{D_{4}}\left(A_{1}, A_{1}, A_{1}, A_{1}\right)=6750 .
\end{aligned}
$$

In total, we obtain

$$
\begin{aligned}
& 1+(45+150+100+75+165+90+15+45+5+18+5) \frac{m}{2}+\left(n_{1,1}+2 n_{2,1}+2 n_{3,1}+n_{2,2}\right)\left(\begin{array}{c}
m / 2 \\
2
\end{array}\right) \\
& +\left(n_{1,1,1}+3 n_{2,1,1}\right)\left(\begin{array}{c}
m / 2 \\
3
\end{array}\right)+n_{1,1,1,1}\left(\begin{array}{c}
m / 2 \\
4
\end{array}\right)=\frac{(5 m+4)(3 m+2)(5 m+2)(15 m+4)}{64}
\end{aligned}
$$

elements in $\operatorname{Fix}_{N C^{m}\left(E_{8}\right)}\left(\phi^{p}\right)$, which agrees with the limit in 6.6g).

\section{CyClic Sieving II}

In this section we present the second cyclic sieving conjecture due to Bessis and Reiner [9, Conj. 6.5].

Let $\psi: N C^{m}(W) \rightarrow N C^{m}(W)$ be the map defined by

$$
\left(w_{0} ; w_{1}, \ldots, w_{m}\right) \mapsto\left(c w_{m} c^{-1} ; w_{0}, w_{1}, \ldots, w_{m-1}\right) .
$$

For $m=1$, we have $w_{0}=c w_{1}^{-1}$, so that this action reduces to the inverse of the Kreweras complement $K_{\text {id }}^{c}$ as defined by Armstrong [2, Def. 2.5.3].

It is easy to see that $\psi^{(m+1) h}$ acts as the identity, where $h$ is the Coxeter number of $W$ (see (8.1) below). By slight abuse of notation as before, let $C_{2}$ be the cyclic group of order $(m+1) h$ generated by $\psi$.

Given these definitions, we are now in the position to state the second cyclic sieving conjecture of Bessis and Reiner. By the results of [19] and of this paper, it becomes the following theorem.

Theorem 33. For an irreducible well-generated complex reflection group $W$ and any $m \geq 1$, the triple $\left(N C^{m}(W), \mathrm{Cat}^{m}(W ; q), C_{2}\right)$, where $\mathrm{Cat}^{m}(W ; q)$ is the q-analogue of the Fuß-Catalan number defined in (3.2), exhibits the cyclic sieving phenomenon. 
By definition of the cyclic sieving phenomenon, we have to prove that

$$
\left|\operatorname{Fix}_{N C^{m}(W)}\left(\psi^{p}\right)\right|=\left.\operatorname{Cat}^{m}(W ; q)\right|_{q=e^{2 \pi i p /(m+1) h}},
$$

for all $p$ in the range $0 \leq p<(m+1) h$.

\section{Auxiliary Results II}

This section collects several auxiliary results which allow us to reduce the problem of proving Theorem 33, respectively the equivalent statement (7.2), for the 26 exceptional groups listed in Section 2 to a finite problem. The corresponding lemmas, Lemmas 34 39, are analogues of Lemmas 26, 28 and 30, 32 in Section 5.

Let $p=a(m+1)+b, 0 \leq b<m+1$. We have

$$
\begin{aligned}
& \psi^{p}\left(\left(w_{0} ; w_{1}, \ldots, w_{m}\right)\right) \\
&=\left(c^{a+1} w_{m-b+1} c^{-a-1} ; c^{a+1} w_{m-b+2} c^{-a-1}, \ldots, c^{a+1} w_{m} c^{-a-1},\right. \\
&\left.c^{a} w_{0} c^{-a}, \ldots, c^{a} w_{m-b} c^{-a}\right) .
\end{aligned}
$$

Lemma 34. It suffices to check (7.2) for $p$ a divisor of $(m+1) h$. More precisely, let $p$ be a divisor of $(m+1) h$, and let $k$ be another positive integer with $\operatorname{gcd}(k,(m+1) h / p)=1$, then we have

$$
\left.\operatorname{Cat}^{m}(W ; q)\right|_{q=e^{2 \pi i p /(m+1) h}}=\left.\operatorname{Cat}^{m}(W ; q)\right|_{q=e^{2 \pi i k p /(m+1) h}}
$$

and

$$
\left|\operatorname{Fix}_{N C^{m}(W)}\left(\psi^{p}\right)\right|=\left|\operatorname{Fix}_{N C^{m}(W)}\left(\psi^{k p}\right)\right| .
$$

Proof. For (8.3), this follows in the same way as (5.3) in Lemma 26.

For (8.2), we must argue differently than in Lemma 26. Let us write $\zeta=e^{2 \pi i p /(m+1) h}$. For a given group $W$, we write $S_{1}(W)$ for the set of all indices $i$ such that $\zeta^{d_{i}-h}=1$, and we write $S_{2}(W)$ for the set of all indices $i$ such that $\zeta^{d_{i}}=1$. By the rule of de l'Hospital, we have

$$
\left.\operatorname{Cat}^{m}(W ; q)\right|_{q=e^{2 \pi i p /(m+1) h}}= \begin{cases}0 & \text { if }\left|S_{1}(W)\right|>\left|S_{2}(W)\right| \\ \frac{\prod_{i \in S_{1}(W)}\left(m h+d_{i}\right)}{\prod_{i \in S_{2}(W)} d_{i}} \frac{\prod_{i \notin S_{1}(W)}\left(1-\zeta^{d_{i}-h}\right)}{\prod_{i \notin S_{2}(W)}\left(1-\zeta^{d_{i}}\right)}, & \text { if }\left|S_{1}(W)\right|=\left|S_{2}(W)\right| .\end{cases}
$$

Since, by Theorem 25, Cat $^{m}(W ; q)$ is a polynomial in $q$, the case $\left|S_{1}(W)\right|<\left|S_{2}(W)\right|$ cannot occur.

We claim that, for the case where $\left|S_{1}(W)\right|=\left|S_{2}(W)\right|$, the factors in the quotient of products

$$
\frac{\prod_{i \notin S_{1}(W)}\left(1-\zeta^{d_{i}-h}\right)}{\prod_{i \notin S_{2}(W)}\left(1-\zeta^{d_{i}}\right)}
$$

cancel pairwise. If we assume the correctness of the claim, it is obvious that we get the same result if we replace $\zeta$ by $\zeta^{k}$, where $\operatorname{gcd}(k,(m+1) h / p)=1$, hence establishing (8.2).

In order to see that our claim is indeed valid, we proceed in a case-by-case fashion, making appeal to the classification of irreducible well-generated complex reflection groups, which we recalled in Section 2. First of all, since $d_{n}=h$, the set $S_{1}(W)$ is always non-empty as it contains the element $n$. Hence, if we want to have $\left|S_{1}(W)\right|=\left|S_{2}(W)\right|$, 
the set $S_{2}(W)$ must be non-empty as well. In other words, the integer $(m+1) h / p$ must divide at least one of the degrees $d_{1}, d_{2}, \ldots, d_{n}$. In particular, this implies that, for each fixed reflection group $W$ of exceptional type, only a finite number of values of $(m+1) h / p$ has to be checked. Writing $M$ for $(m+1) h / p$, what needs to be checked is whether the multisets (that is, multiplicities of elements must be taken into account)

$$
\left\{\left(d_{i}-h\right) \bmod M: i \notin S_{1}(W)\right\} \quad \text { and } \quad\left\{d_{i} \bmod M: i \notin S_{2}(W)\right\}
$$

are the same. Since, for a fixed irreducible well-generated complex reflection group, there is only a finite number of possibilities for $M$, this amounts to a routine verification.

Lemma 35. Let $p$ be a divisor of $(m+1) h$. If $p$ is divisible by $m+1$, then (17.2) is true.

We leave the proof to the reader as it is completely analogous to the proof of Lemma 27.

Lemma 36. Equation (7.2) holds for all divisors $p$ of $m+1$.

Proof. We have

$$
\left.\mathrm{Cat}^{m}(W ; q)\right|_{q=e^{2 \pi i p /(m+1) h}}= \begin{cases}0 & \text { if } p<m+1 \\ m+1 & \text { if } p=m+1\end{cases}
$$

Here, the first case follows from (8.4) and the fact that we have $S_{1}(W) \supseteq\{n\}$ and $S_{2}(W)=\emptyset$ if $p \mid(m+1)$ and $p<m+1$.

On the other hand, if $\left(w_{0} ; w_{1}, \ldots, w_{m}\right)$ is fixed by $\psi^{p}$, then one can apply an argument similar to that in Lemma 28 with any $w_{i}$ taking the role of $w_{1}, 0 \leq i \leq m$. It follows that if $p=m+1$, the set $\operatorname{Fix}_{N C^{m}(W)}\left(\psi^{p}\right)$ consists of the $m+1$ elements $\left(w_{0} ; w_{1}, \ldots, w_{m}\right)$ obtained by choosing $w_{i}=c$ for a particular $i$ between 0 and $m$, all other $w_{j}$ 's being equal to $\varepsilon$. If $p<m+1$, then there is no element in $\operatorname{Fix}_{N C^{m}(W)}\left(\psi^{p}\right)$.

Lemma 37. Let $W$ be an irreducible well-generated complex reflection group of rank $n$, and let $p=m_{1} h_{1}$ be a divisor of $(m+1) h$, where $m+1=m_{1} m_{2}$ and $h=h_{1} h_{2}$. We assume that $\operatorname{gcd}\left(h_{1}, m_{2}\right)=1$. Suppose that Theorem 33 has already been verified for all irreducible well-generated complex reflection groups with rank $<n$. If $h_{2}$ does not divide all degrees $d_{i}$, then equation (17.2) is satisfied.

We leave the proof to the reader as it is completely analogous to the proof of Lemma 30.

Lemma 38. Let $W$ be an irreducible well-generated complex reflection group of rank $n$, and let $p=m_{1} h_{1}$ be a divisor of $(m+1) h$, where $m+1=m_{1} m_{2}$ and $h=h_{1} h_{2}$. We assume that $\operatorname{gcd}\left(h_{1}, m_{2}\right)=1$. If $m_{2}>n$ then

$$
\operatorname{Fix}_{N C^{m}(W)}\left(\psi^{p}\right)=\emptyset \text {. }
$$

We leave the proof to the reader as it is analogous to the proof of Lemma 31 .

Remark 4. By applying the same reasoning as in Remark 3 with Lemmas 30 and 31 replaced by Lemmas 37 and 38, respectively, it follows that we only need to check (7.2) for pairs $\left(m_{2}, h_{2}\right)$ satisfying (5.9) and $m_{2} \leq n$. This reduces the problem to a finite number of choices. 
Lemma 39. Let $W$ be an irreducible well-generated complex reflection group of rank $n$ with the property that $d_{i} \mid h$ for $i=1,2, \ldots, n$. Then Theorem 33 is true for this group $W$.

Proof. Proceeding in a fashion analogous to the beginning of the proof of Lemma 32, we may restrict to the case where $p \mid(m+1) h$ and $(m+1) h / p$ does not divide any of the $d_{i}$ 's. In this case, it follows from (8.4) and the fact that we have $S_{1}(W) \supseteq\{n\}$ and $S_{2}(W)=\emptyset$ that the right-hand side of (7.2) equals 0 . Inspection of the classification of all irreducible well-generated complex reflection groups, which we recalled in Section 2 , reveals that all groups satisfying the hypotheses of the lemma have rank $n \leq 2$. Except for the groups contained in the infinite series $G(d, 1, n)$ and $G(e, e, n)$ for which Theorem 2 has been established in [19], these are the groups $G_{5}, G_{6}, G_{9}, G_{10}, G_{14}, G_{17}, G_{18}, G_{21}$. The verification of (7.2) can be done in a similar fashion as in the proof of Lemma 32. We illustrate this by going through the case of the group $G_{6}$. In analogy with the earlier situation, we note that Lemma 38 implies that equation (7.2) holds if $m_{2}>2$, so that in the following arguments we may assume that $m_{2}=2$.

CASE $G_{6}$. The degrees are 4,12, and therefore, according to Remark 4, we need only consider the case where $h_{2}=4$ and $m_{2}=2$, that is, $p=3(m+1) / 2$. Then the action of $\psi^{p}$ is given by

$$
\psi^{p}\left(\left(w_{0} ; w_{1}, \ldots, w_{m}\right)\right)=\left(c^{2} w_{\frac{m+1}{2}} c^{-2} ; c^{2} w_{\frac{m+3}{2}} c^{-2}, \ldots, c^{2} w_{m} c^{-2}, c w_{0} c^{-1}, \ldots, c w_{\frac{m-1}{2}} c^{-1}\right) .
$$

If $\left(w_{0} ; w_{1}, \ldots, w_{m}\right)$ is fixed by $\psi^{p}$, there must exist an $i$ with $0 \leq i \leq \frac{m-1}{2}$ such that $\ell_{T}\left(w_{i}\right)=1, w_{i} c w_{i} c^{-1}=c$, and all $w_{j}, j \neq i, \frac{m+1}{2}+i$, equal $\varepsilon$. However, with the help of CHEVIE, one verifies that there is no such solution to this equation. Hence, the left-hand side of (7.2) is equal to 0 , as required.

This completes the proof of the lemma.

\section{EXEMPlificAtion OF CASE-BY-CASE VERIFICATION OF THEOREM 33}

It remains to verify Theorem 33 for the groups $G_{4}, G_{8}, G_{16}, G_{20}, G_{23}=H_{3}, G_{24}, G_{25}$, $G_{26}, G_{27}, G_{28}=F_{4}, G_{29}, G_{30}=H_{4}, G_{32}, G_{33}, G_{34}, G_{35}=E_{6}, G_{36}=E_{7}, G_{37}=E_{8}$. All details can be found in [21, Sec. 9]. We content ourselves with discussing the case of the group $G_{24}$, as this suffices to convey the flavour of the necessary computations.

In order to simplify our considerations, it should be observed that the action of $\psi$ (given in (7.1) ) is exactly the same as the action of $\phi$ (given in (3.1)) with $m$ replaced by $m+1$ on the components $w_{1}, w_{2}, \ldots, w_{m+1}$, that is, if we disregard the 0 -th component of the elements of the generalised non-crossing partitions involved. The only difference which arises is that, while the $(m+1)$-tuples $\left(w_{0} ; w_{1}, \ldots, w_{m}\right)$ in (7.1) must satisfy $w_{0} w_{1} \cdots w_{m}=c$, for $w_{1}, w_{2}, \ldots, w_{m+1}$ in (3.1) we only must have $w_{1} w_{2} \cdots w_{m+1} \leq_{T} c$. Consequently, we may use the counting results from Section 6. except that we have to restrict our attention to those elements $\left(w_{0} ; w_{1}, \ldots, w_{m}, w_{m+1}\right) \in N C^{m+1}(W)$ for which $w_{1} w_{2} \cdots w_{m+1}=c$, or, equivalently, $w_{0}=\varepsilon$.

CAse $G_{24}$. The degrees are 4,6,14, and hence we have

$$
\operatorname{Cat}^{m}\left(G_{24} ; q\right)=\frac{[14 m+14]_{q}[14 m+6]_{q}[14 m+4]_{q}}{[14]_{q}[6]_{q}[4]_{q}}
$$


Let $\zeta$ be a $14(m+1)$-th root of unity. The following cases on the right-hand side of (7.2) occur:

$$
\begin{aligned}
& \lim _{q \rightarrow \zeta} \operatorname{Cat}^{m}\left(G_{24} ; q\right)=m+1, \quad \text { if } \zeta=\zeta_{14}, \zeta_{7}, \\
& \lim _{q \rightarrow \zeta} \operatorname{Cat}^{m}\left(G_{24} ; q\right)=\frac{7 m+7}{3}, \quad \text { if } \zeta=\zeta_{6}, \zeta_{3}, 3 \mid(m+1), \\
& \lim _{q \rightarrow \zeta} \operatorname{Cat}^{m}\left(G_{24} ; q\right)=\operatorname{Cat}^{m}\left(G_{24}\right), \quad \text { if } \zeta=-1 \text { or } \zeta=1, \\
& \lim _{q \rightarrow \zeta} \operatorname{Cat}^{m}\left(G_{24} ; q\right)=0, \quad \text { otherwise. }
\end{aligned}
$$

We must now prove that the left-hand side of (7.2) in each case agrees with the values exhibited in (9.1). The only cases not covered by Lemma 35 are the ones in (9.1b) and (9.1d). On the other hand, the only cases left to consider according to Remark 4 are the cases where $h_{2}=1$ and $m_{2}=3, h_{2}=2$ and $m_{2}=3$, and $h_{2}=m_{2}=2$. These correspond to the choices $p=14(m+1) / 3, p=7(m+1) / 3$, respectively $p=7(m+1) / 2$. The first two cases belong to (9.1b), while $p=7(m+1) / 2$ belongs to $(9.1 \mathrm{~d})$.

In the case that $p=7(m+1) / 3$, the action of $\psi^{p}$ is given by

$$
\begin{aligned}
\psi^{p}\left(\left(w_{0} ; w_{1}, \ldots,\right.\right. & \left.\left.w_{m}\right)\right) \\
& =\left(c^{3} w_{\frac{2 m+2}{3}} c^{-3} ; c^{3} w_{\frac{2 m+5}{3}} c^{-3}, \ldots, c^{3} w_{m} c^{-3}, c^{2} w_{0} c^{-2}, \ldots, c^{2} w_{\frac{2 m-1}{3}} c^{-2}\right) .
\end{aligned}
$$

Hence, for an $i$ with $0 \leq i \leq \frac{m-2}{3}$, we must find an element $w_{i}=t_{1}$, where $t_{1}$ satisfies (6.4), so that we can set $w_{i+\frac{m+1}{3}}=c^{2} t_{1} c^{-2}, w_{i+\frac{2 m+2}{3}}=c^{4} t_{1} c^{-4}$, and all other $w_{j}$ 's equal to $\varepsilon$. We have found seven solutions to the counting problem (6.4), and each of them gives rise to $(m+1) / 3$ elements in $\operatorname{Fix}_{N C^{m}\left(G_{24}\right)}\left(\psi^{p}\right)$ since the index $i$ ranges from 0 to $(m-2) / 3$.

On the other hand, if $p=14(m+1) / 3$, then the action of $\psi^{p}$ is given by

$$
\begin{aligned}
& \psi^{p}\left(\left(w_{0} ; w_{1}, \ldots, w_{m}\right)\right) \\
& \quad=\left(c^{5} w_{\frac{m+1}{3}} c^{-5} ; c^{5} w_{\frac{m+4}{3}} c^{-5}, \ldots, c^{5} w_{m} c^{-5}, c^{4} w_{0} c^{-4}, \ldots, c^{4} w_{\frac{m-2}{3}} c^{-4}\right) .
\end{aligned}
$$

By Lemma 29, every element of $N C(W)$ is fixed under conjugation by $c^{7}$, and, thus, the equations for $t_{1}$ in this case are the same as in the previous one where $p=7(m+1) / 3$.

Hence, in either case, we obtain $7 \frac{m+1}{3}=\frac{7 m+7}{3}$ elements in $\operatorname{Fix}_{N C^{m}\left(G_{24}\right)}\left(\psi^{p}\right)$, which agrees with the limit in $9.1 \mathrm{~b}$ ).

If $p=7(m+1) / 2$, the relevant counting problem is (6.5). However, no element $\left(w_{0} ; w_{1}, \ldots, w_{m}\right) \in \operatorname{Fix}_{N C^{m}\left(G_{24}\right)}\left(\psi^{p}\right)$ can be produced in this way since the counting problem imposes the restriction that $\ell_{T}\left(w_{0}\right)+\ell_{T}\left(w_{1}\right)+\cdots+\ell_{T}\left(w_{m}\right)$ be even, which contradicts the fact that $\ell_{T}(c)=n=3$. This is in agreement with the limit in (9.1d).

\section{ACKNOWLEDGEMENTS}

The authors thank an anonymous referee for a very careful reading of the original manuscript, and for numerous pertinent suggestions which have helped to considerably improve the original manuscript. 


\section{REFERENCES}

[1] G. E. Andrews, The Theory of Partitions, Encyclopedia of Math. and its Applications, vol. 2, Addison-Wesley, Reading, 1976.

[2] D. Armstrong, Generalized noncrossing partitions and combinatorics of Coxeter groups, Mem. Amer. Math. Soc., vol. 202, no. 949, Amer. Math. Soc., Providence, R.I., 2009.

[3] D. Armstrong, C. Stump and H. Thomas, A uniform bijection between nonnesting and noncrossing partitions, Trans. Amer. Math. Soc. (to appear).

[4] C. A. Athanasiadis, Generalized Catalan numbers, Weyl groups and arrangements of hyperplanes, Bull. London Math. Soc. 36 (2004), 294-302.

[5] C. A. Athanasiadis, On a refinement of the generalized Catalan numbers for Weyl groups, Trans. Amer. Math. Soc. 357 (2005), 179-196.

[6] D. Bessis, The dual braid monoid, Ann. Sci. École Norm. Sup. (4) 36 (2003), 647-683.

[7] D. Bessis, Finite complex reflection groups are $K(\pi, 1)$, preprint, ar $\chi \mathrm{iv}:$ math/0610777.

[8] D. Bessis and R. Corran, Non-crossing partitions of type (e,e,r), Adv. Math. 202 (2006), 1-49.

[9] D. Bessis and V. Reiner, Cyclic sieving and noncrossing partitions for complex reflection groups, Ann. Comb. 15 (2011), 197-222.

[10] T. Brady and C. Watt, $K(\pi, 1)$ 's for Artin groups of finite type, Geom. Dedicata 94 (2002), $225-250$.

[11] F. Chapoton, Enumerative properties of generalized associahedra, Séminaire Lotharingien Combin. 51 (2004), Article B51b, 16 pp.

[12] P. Edelman, Chain enumeration and noncrossing partitions, Discrete Math. 31 (1981), 171-180.

[13] S. Fomin and N. Reading, Generalized cluster complexes and Coxeter combinatorics, Int. Math. Res. Notices 44 (2005), 2709-2757.

[14] M. Geck, G. Hiss, F. Lübeck, G. Malle and G. Pfeiffer, CHEVIE - a system for computing and processing generic character tables for finite groups of Lie type, Appl. Algebra Engrg. Comm. Comput. 7 (1996), 175-210.

[15] I. Gordon and S. Griffeth, Catalan numbers for complex reflection groups, Amer. J. Math. (to appear).

[16] J. E. Humphreys, Reflection groups and Coxeter groups, Cambridge University Press, Cambridge, 1990.

[17] C. Krattenthaler, The F-triangle of the generalised cluster complex, in: Topics in Discrete Mathematics, dedicated to Jarik Nešetřil on the occasion of his 60th birthday, M. Klazar, J. Kratochvil, M. Loebl, J. Matoušek, R. Thomas and P. Valtr (eds.), Springer-Verlag, Berlin, New York, 2006, pp. 93-126.

[18] C. Krattenthaler, The $M$-triangle of generalised non-crossing partitions for the types $E_{7}$ and $E_{8}$, Séminaire Lotharingien Combin. 54 (2006), Article B54l, 34 pages.

[19] C. Krattenthaler, Non-crossing partitions on an annulus, in preparation.

[20] C. Krattenthaler and T. W. Müller, Decomposition numbers for finite Coxeter groups and generalised non-crossing partitions, Trans. Amer. Math. Soc. 362 (2010), 2723-2787.

[21] C. Krattenthaler and T. W. Müller, Cyclic sieving for generalised non-crossing partitions associated with complex reflection groups of exceptional type - the details, manuscript; ar $\chi$ iv: 1001.0030.

[22] G. Kreweras, Sur les partitions non croisées d'un cycle, Discrete Math. 1 (1972), 333-350.

[23] G. I. Lehrer and J. Michel, Invariant theory and eigenspaces for unitary reflection groups, C. R. Math. Acad. Sci. Paris 336 (2003), 795-800.

[24] G. I. Lehrer and D. E. Taylor, Unitary reflection groups, Cambridge University Press, Cambridge, 2009.

[25] N. A. Loehr, Conjectured statistics for the higher q, t-Catalan sequences, Electron. J. Combin. 12 (2005), Art. \#R9, 54 pp.

[26] G. Malle and J. Michel, Constructing representations of Hecke algebras for complex reflection groups, LMS J. Comput. Math. 13 (2010), 426-450.

[27] I. Marin, The cubic Hecke algebra on at most 5 strands, preprint, ar $\chi$ iv:1110.6621.

[28] J. Michel, The GAP-part of the CHEVIE system, GAP 3-package available for download from http://people.math.jussieu.fr/jmichel/chevie/chevie.html. 
[29] P. Orlik and L. Solomon, Unitary reflection groups and cohomology, Invent. Math. 59 (1980), $77-94$.

[30] V. Reiner, D. Stanton and D. White, The cyclic sieving phenomenon, J. Combin. Theory Ser. A 108 (2004), 17-50.

[31] V. Ripoll, Orbites d'Hurwitz des factorisations primitives d'un élément de Coxeter, J. Algebra 323 (2010), 1432-1453.

[32] G. C. Shephard and J. A. Todd, Finite unitary reflection groups, Canad. J. Math. 6 (1954), 274-304.

[33] T. A. Springer, Regular elements of finite reflection groups, Invent. Math. 25 (1974), 159-198.

[34] J. R. Stembridge, Some hidden relations involving the ten symmetry classes of plane partitions, J. Combin. Theory Ser. A 68 (1994), 372-409.

[35] J. R. Stembridge, Canonical bases and self-evacuating tableaux, Duke Math. J. 82 (1996). 585-606,

[36] J. R. Stembridge, coxeter, Maple package for working with root systems and finite Coxeter groups; available at http://www.math.lsa.umich.edu/ jos

Fakultät für Mathematik, Universität Wien, Nordbergstrasse 15, A-1090 Vienna, AustRIA. WWW: http://wWw.mat.univie.ac.at/ kratt.

School of Mathematical Sciences, Queen Mary \& Westfield College, University of London, Mile End Road, London E1 4NS, United Kingdom.

http://www.maths.qmw.ac.uk/ twm/. 